\title{
Measuring precession in asymmetric compact binaries
}

\author{
Geraint Pratten $\odot,{ }^{*}$ Patricia Schmidt $\odot,{ }^{\dagger}$ Riccardo Buscicchio $\odot,{ }^{\ddagger}$ and Lucy M. Thomas $\odot^{\S}$ \\ School of Physics and Astronomy and Institute for Gravitational Wave Astronomy, University of Birmingham, Edgbaston, \\ Birmingham B15 2TT, United Kingdom
}

(Received 30 June 2020; accepted 28 September 2020; published 19 October 2020)

\begin{abstract}
Gravitational-wave observations of merging compact binaries hold the key to precision measurements of the objects' masses and spins. General relativistic precession, caused by spins misaligned with the orbital angular momentum, is considered a crucial tracer for determining the binary's formation history and environment and it also improves mass estimates; its measurement is therefore of particular interest with wide-ranging implications. Precession leaves a characteristic signature in the emitted gravitational-wave signal that is even more pronounced in binaries with highly unequal masses. The recent observations of GW190412 and GW190814 have confirmed the existence of such asymmetric compact binaries. Here we perform a systematic study to assess the confidence in measuring precession in gravitational-wave observations of high-mass-ratio binaries and our ability to measure the mass of the lighter companion in neutron-star-black-hole-type systems. Using Bayesian model selection, we show that precession can be decisively identified for low-mass binaries with mass ratios as low as 1:3 and mildly precessing spins with magnitudes $\lesssim 0.4$, even in the presence of systematic waveform errors.
\end{abstract}

DOI: 10.1103/PhysRevResearch.2.043096

\section{INTRODUCTION}

The LIGO Scientific and Virgo collaborations have recently reported the first clear detections of gravitational waves (GWs) from coalescing compact binaries with unequal masses, GW190412 [1] and GW190814 [2]. The inferred mass ratio ${ }^{1} q=m_{1} / m_{2} \geqslant 1$ for both events points to highly asymmetric compact binary systems, differing from the binary black holes observed during the first two observing runs $\mathrm{O} 1$ [3] and O2 [4]. Of these two events, GW190412 is consistent with a binary-black-hole (BBH) merger, with a primary source mass of $m_{1} \simeq 30 M_{\odot}$ and a secondary source mass of $m_{2} \simeq 8 M_{\odot}$. The second event GW190814 is consistent with the merger of a neutron star (NS) and black hole (BH) or a black hole and binary [2], with a primary source mass of $m_{1} \simeq 23 M_{\odot}$ and a secondary mass $m_{2} \simeq 2.6 M_{\odot}$. Notably, the mass of the secondary lies in the lower mass gap of $2.5 M_{\odot}-5 M_{\odot}$ [5-9], making it either the heaviest NS or the lightest BH observed to date [6-17]. A coincident observation of an electromagnetic (EM) counterpart, such as a $\gamma$-ray burst or a kilonova, would indicate strongly that the lighter compact object was a neutron star [18-28]. Alternatively, we may hope

\footnotetext{
*gpratten@star.sr.bham.ac.uk

†pschmidt@star.sr.bham.ac.uk

†riccardo@star.sr.bham.ac.uk

§1thomas@star.sr.bham.ac.uk

${ }^{1}$ Note that we adopt the inverse convention to that used in $[1,2]$, where $q=m_{2} / m_{1}$.

Published by the American Physical Society under the terms of the Creative Commons Attribution 4.0 International license. Further distribution of this work must maintain attribution to the author(s) and the published article's title, journal citation, and DOI.
}

to see the tidal disruption of the NS in an NSBH system [22,29-41], which would leave a characteristic imprint in the emitted GW signal. However, as the mass ratio increases, tidal effects become highly suppressed and the NS can be swallowed entirely before tidal disruption has taken place, making a highly asymmetric NSBH merger indistinguishable from a BBH merger [36]. In addition, tidal effects in the early inspiral are anticipated to be negligible for such high-massratio binaries and we are therefore reliant on the measurement of other intrinsic parameters such as masses and spins to determine the binary composition. Accurate measurements of the component masses and spins will be important to discriminate as to whether the lighter compact object is consistent with the theoretical limits on NS masses [8] or with a low-mass BH [7].

While the formation of compact binaries with asymmetric mass ratios is highly uncertain, such unequal-mass binaries are of particular interest for measuring relativistic spin effects such as spin precession. Spin precession is sourced by the misalignment between the orbital angular momentum of the binary motion and the individual spins of the two compact objects, inducing additional structure in the form of characteristic modulations in the GW signal $[42,43]$. This helps breaking correlations such as the mass-spin degeneracy, in which one can mimic the effect of spin by modifying the mass ratio of the compact binary. By breaking these degeneracies, we can infer tighter mass constraints [44-49]. Moreover, the orientation of the spin angular momenta is considered one of the main tracers of a binary's formation channel and may help in discerning the nature of the compact objects [50-64]. Gravitational wave observations so far, however, have not yielded a confident measurement of precession effects, with the events observed to date being consistent with either small spins or large misaligned spins [1,2,4]. GW190814 
provided the tightest constraint on precession from all $\mathrm{GW}$ observations to date and constrained precession to be near zero [2].

In this paper, we reassess the confidence to which we can measure spin-precession effects in high-mass-ratio binaries similar to GW190814 and the confidence to which we can constrain the mass of the lighter companion at current detector sensitivity. First, we investigate the degree to which we can constrain precession in GW observations of asymmetric binaries using Bayesian model selection and several statistical measures. Second, we demonstrate the efficacy of spin precession in breaking the mass-spin degeneracy in order to improve constraints on the mass of the secondary companion [44-47]. We use simulated GW signals from moderately inclined binaries with different mass ratios and varying amount of precession to study the measurability of precession effects in a systematic way. We demonstrate that even small amounts of precession can be identified confidently despite the presence of systematic errors. Further, using a second set of simulated signals with the smaller object between $1.25 M_{\odot}$ and $3 M_{\odot}$, we find that the mass of the secondary is consistently underestimated for the binaries considered when spin precession is neglected, leading to an increased risk of misidentifying a low-mass BH as a NS. Similar questions focusing on large populations have been addressed in previous studies [65-67]. Here we focus primarily on GW190814-like binaries, adopting masses, inclinations, and signal-to-noise ratios (SNRs) broadly consistent with the values reported in [2]. We note that the results presented in Sec. IV A are also broadly applicable to binaries similar to GW190412.

This paper is organized as follows. In Sec. II we provide an introduction to asymmetric binaries and precession. We then introduce our methodology in Sec. III, before presenting our main results in Sec. IV. We discuss our results and summarize in Sec. V. Throughout this paper we set $G=c=1$ unless stated otherwise.

\section{ASYMMETRIC COMPACT BINARIES}

The current understanding of binary evolution leads to a number of distinct binary formation channels. Proposed scenarios include isolated [68-71], dynamical [72-80], and primordial [81,82] formation with many subchannels within each category. Each of these formation channels will leave a characteristic imprint on the mass [62,83-86], spin [51,54,59,61], and redshift [87-93] distributions of the observed compact binaries.

Modeling of the mass distribution using the ten BBHs detected during the first two observing runs [4] finds a median mass ratio of $q=1.1$ at $90 \%$ credibility and predicts that $99 \%$ of binaries detected will have mass ratios $q<2$ [94]. This makes the recent observation of GW190814, a highly asymmetric binary with a mass ratio of $q \sim 9$, something of an enigma. Plausible formation channels for such asymmetric binaries include dynamical [95,96] and hierarchical merger scenarios $[56,58,80,97,98]$. For isolated binary formation channels, the prevalence of asymmetric compact binaries can be sensitive to the metallicity of the environment, with asymmetric binaries being preferred in low-metallicity environments $[71,99,100]$. Accretion disks of active galactic nuclei could be promising environments for driving hierarchical mergers, in which asymmetric binaries are likely [101].

Furthermore, while the primary mass allows us to identify the heavier component as a black hole, the secondary mass is compatible with being either a $\mathrm{BH}$ or a NS. We note, however, that the lighter companion with $\sim 2.6 M_{\odot}$ is at the threshold of the maximum theoretically supported NS mass [14,102-104] and is in tension with current constraints from the maximum NS masses inferred from GW170817 and pulsar observations [10,14-16]. In addition, the mass of the secondary is comparable to the $\mathrm{BH}$ masses created as binary-neutron-star merger products $[14,105,106]$ as well as a recently reported low-mass $\mathrm{BH}$ in a noninteracting $\mathrm{BH}-$ giant-star binary [107]. Population synthesis models for the formation of NSBH binaries demonstrate a preference for a system comprising a heavy NS $\left(m_{\mathrm{NS}} \sim 1.3 M_{\odot}-2.0 M_{\odot}\right)$ and a low-mass $\mathrm{BH}\left(m_{\mathrm{BH}} \sim 5 M_{\odot}-15 M_{\odot}\right)$, especially for formation channels with low natal kicks [108]. Such binaries would correspond to mass ratios $q \sim 3-8$, further emphasizing the need to understand the confidence to which we can infer the intrinsic properties of asymmetric binaries from $\mathrm{GW}$ observations.

Another source of asymmetry, besides unequal masses, pertains to the spins of the two companions, which are of particular interest for discriminating between different formation channels. Binaries that form through dynamical interactions are anticipated to have isotropically oriented spins. This is in stark contrast to binaries that form from isolated compact objects, where spins are preferentially aligned with the orbital angular momentum. For isolated binaries, supernova kicks are one of the primary mechanisms that give rise to spins misaligned with the orbital angular momenta [50]. Constraints on precession in compact binaries can therefore significantly shape our understanding of binary formation channels and their evolution.

In order to infer the source properties from $\mathrm{GW}$ observations, highly accurate waveform models that govern the inspiral, merger, and ringdown are necessary. The GW signals of compact binaries with highly asymmetric masses possess a rich phenomenology due to the excitation of higher-order multipoles. The higher-order modes of the gravitational field encode additional information about the source which allows for the breaking of certain parameter degeneracies, such as the inclination-distance correlation [109-111].

Binaries whose spins are aligned with the orbital angular momentum exhibit strong correlations between the masses/mass ratio and spins [112]. Arbitrarily oriented spins, however, break the equatorial symmetry of the binary system and induce general relativistic spin precession [42,43,113,114]. These affect the emitted signal in several ways: (i) They leave characteristic imprints in the form of amplitude and phase modulations; (ii) they modify the final state of the remnant; (iii) they excite higher-order modes. Similar to unequal masses, precession of the orbital plane allows us to break another parameter correlation, the massspin degeneracy $[44,45,47]$. Spin precession could therefore be of particular importance when one seeks to distinguish between NSs and low-mass BHs in the absence of a clear tidal signature. 
In what follows, we will be considering simulated signals that contain both higher-order modes and precession in order to mimic a realistic scenario as best as possible.

\section{METHODOLOGY}

\section{A. Effective precession spin}

Coalescing BBHs on quasispherical orbits are intrinsically characterized by their mass ratio $q=m_{1} / m_{2} \geqslant 1$, where $m_{i}$ is the component mass of the $i$ th black hole, and their (dimensionless) spin angular momenta $\vec{\chi}_{i}$. The dominant spin effect on the inspiral rate is captured by the effective aligned spin, a mass-weighted combination of the spins parallel to the orbital angular momentum $\hat{L}$ [115-117],

$$
\chi_{\mathrm{eff}}=\frac{m_{1} \chi_{1}+m_{2} \chi_{2}}{m_{1}+m_{2}} .
$$

Depending on the binary's formation history, however, the spins may be arbitrarily oriented with respect to the orbital angular momentum $\hat{L}$ [54]. Misalignment between the spins and $\hat{L}$ induces general relativistic precession of the orbital plane and spins $[42,43]$, i.e., the (four) spin components perpendicular to $\hat{L}$ source these precession effects. Over many GW cycles, these spin components contained within the instantaneous orbital plane may be approximated by a scalar quantity $\chi_{p}$, which captures the average amount of precession in a binary system [118] defined as

$$
\chi_{p}:=\frac{1}{A_{1} m_{1}^{2}} \max \left(A_{1} S_{1 \perp}, A_{2} S_{2 \perp}\right),
$$

where $\vec{S}_{i}=m_{i}^{2} \vec{\chi}_{i}, S_{i \perp}=\left\|\hat{L} \times\left(\vec{S}_{i} \times \hat{L}\right)\right\|, A_{1}=2+3 q / 2$, and $A_{2}=2+3 / 2 q$. The effective precession spin $\chi_{p}$ is defined in the domain $[0,1]$, where $\chi_{p}=0$ corresponds to a nonprecessing and $\chi_{p}=1$ to a maximally precessing binary. It is important to note, however, that even very strongly precessing binaries may not be easily identified as such if the line of sight is approximately along the direction of the total angular momentum, as the imprint of precession on the GW signal will be minimized [119]. We will use the effective precession spin $\chi_{p}$ in our analyses to characterize the amount of precession present in a binary system, and statements concerning the measurability of precession will be based on its inferred distribution.

\section{B. Precessing SNR}

The strength of an observed GW signal $h$ is characterized by its $\operatorname{SNR} \rho$ defined as

$$
\rho:=\sqrt{\langle h \mid h\rangle}=2\left[\int_{0}^{\infty} d f \frac{|\tilde{h}(f)|^{2}}{S_{n}(f)}\right]^{1 / 2},
$$

where $\tilde{h}(f)$ denotes the Fourier transform of $h$ and $S_{n}(f)$ is the noise power spectral density. Recently, Ref. [120] introduced a frequentist framework to estimate the contribution to the SNR $\rho$ that stems from precession, referred to as the precessing SNR $\rho_{p}$. The formalism decomposes the GW signal into two harmonics, each of which is equivalent to the emission of a nonprecessing binary. The modulations typical for a precessing system are introduced through the beating between the two harmonics. Then $\rho_{p}$ is defined as the SNR contained in the harmonic orthogonal to the dominant one. In the absence of precession, $\rho_{p}$ is $\chi^{2}$ distributed with two degrees of freedom. A simple criterion for precession to be considered observable is the requirement that $\rho_{p} \geqslant 2.1[120,121]$. Here we will assess the significance of $\rho_{p}$ via the single-sided $p$ value associated with the mean of the distribution.

The two-harmonic formalism relies on several assumptions [120], which are valid for the signals considered in this paper. We will thus use it as a complementary quantifier to assess the measurability of spin precession. We stress, however, that $\rho_{p}$ is an inherently frequentist quantity, while our main analyses will be fully Bayesian as discussed in Sec. III D.

\section{Simulated gravitational-wave signals}

We create two sets of simulated GW signals (injections), which include both precession and a subset of higher-order modes as expected for real signals. We inject the signals into zero noise, which is representative of the results when averaging over identical injections in different Gaussian noise realizations. All mock signals used in our analyses are generated from the effective-one-body (EOB) waveform model SEOBNRv4PHM [122] for binary black holes. ${ }^{2}$ The EOB framework [123-126] models the complete inspiral-mergerringdown $\mathrm{GW}$ signal of coalescing compact binaries in the time domain. It utilizes analytical information from postNewtonian theory and gravitational self-force and is tuned to numerical relativity (NR) in the strong-field regime. We note that neither the EOB model nor the recovery waveforms described in Sec. III D are calibrated against precessing NR simulations. Precessing NR simulations at high mass ratios are numerically challenging, leading to a lack of waveforms in this region of the binary parameter space. We therefore use SEOBNRv4PHM as our injection model as it incorporates full spin degrees of freedom and higher-order modes and is demonstrably robust at high mass ratios, which is crucial for our study.

The first set of injections has a varying mass ratio $q \in$ $[3,10]$ and $\chi_{p} \in[0.0,0.4]$ chosen such that the lighter companion is always nonspinning. All other parameters are fixed and listed in Table I. They are chosen to be consistent with GW190814 [2], in particular the source frame chirp mass $\mathcal{M}_{c}=\left(m_{1} m_{2}\right)^{3 / 5} /\left(m_{1}+m_{2}\right)^{1 / 5}$ and the inclination. Consistent with the majority of observed signals to date [4], we only consider binaries with a vanishing inspiral spin $\chi_{\text {eff }}$ [128-130]. We do not expect this particular choice to affect our results due to the approximate decoupling between the inspiral and precession dynamics [119,131].

Furthermore, our injections have a fixed SNR of $\rho=30$, representing moderately loud signals at current and nearfuture detector sensitivities [132]. For mass ratio $q=9$ we create additional injections with $\rho=10$ and 20; since we fix the binary inclination to a moderate value of $\sim 40^{\circ}$, this

\footnotetext{
${ }^{2}$ This waveform model does not contain tidal effects, which are negligible for the high mass ratios considered in our analysis. Tidal disruption could in principle occur for some of the lower-mass-ratio binaries but is not taken into account here.
} 
TABLE I. Fixed parameter values for all simulated GW signals in the first data set. The mass-spin degeneracy data set has the same extrinsic parameters, $\mathrm{SNR}$, and $\chi_{\text {eff }}$ but fixes $\chi_{p}=0.2$ and varies $\mathcal{M}_{c}$ instead (see the text). These values are consistent with the parameters inferred for GW190814 [2,127].

\begin{tabular}{lc}
\hline \hline Parameter & Value \\
\hline Chirp mass $\mathcal{M}_{c}\left(M_{\odot}\right)$ & 6.3 \\
Effective inspiral spin $\chi_{\text {eff }}$ & 0.0 \\
Inclination $\iota(\mathrm{rad})$ & 0.70 \\
RA $\alpha(\mathrm{rad})$ & 0.23 \\
DEC $\delta(\mathrm{rad})$ & -0.42 \\
Polarization $\psi(\mathrm{rad})$ & 3.0 \\
SNR $\rho$ & 30 \\
\hline \hline
\end{tabular}

amounts to changing the luminosity distance to adjust the SNR.

A second set of injections explores the mass-spin degeneracy briefly discussed in Sec. II. Here we pin the mass of the primary to $m_{1}=20 M_{\odot}$ and vary the mass of the secondary in the range $m_{2} \in[1.25,1.5, \ldots, 3] M_{\odot}$. The effective inspiral spin is fixed at $\chi_{\text {eff }}=0$ and we allow for small nonvanishing spin precession with $\chi_{p}=0.2$. All other parameters are identical to the first set. This series is chosen to span a range of astrophysically interesting component masses that graze the lower boundary of the mass gap and serves to highlight the importance of precessing waveform models in constraining the component masses, especially near the maximum theoretical NS mass.

\section{Bayesian inference and model selection}

We treat the measurability of precession in an asymmetric binary system as a Bayesian model selection problem. The probability of obtaining the binary parameters $\theta$ given the data $d$ and a signal model hypothesis $\mathcal{H}$ is

$$
p(\theta \mid d, \mathcal{H})=\frac{\mathcal{L}(d \mid \theta, \mathcal{H}) \pi(\theta \mid \mathcal{H})}{\mathcal{Z}_{\mathcal{H}}},
$$

where $\mathcal{L}$ is the likelihood, $\pi$ the prior, and $\mathcal{Z}$ the signal evidence,

$$
\mathcal{Z}_{\mathcal{H}} \equiv \int d \theta \mathcal{L}(d \mid \mathcal{H}, \theta) \pi(\theta \mid \mathcal{H}),
$$

such that the noise evidence $\mathcal{Z}_{n}$ is defined by

$$
\mathcal{Z}_{n} \equiv \mathcal{L}(d \mid n)
$$

The Bayes factor $\mathcal{B}$ for a signal, assuming a model hypothesis $\mathcal{H}$, over noise $n$ is

$$
\mathcal{B}_{\mathcal{H} / n}=\frac{\mathcal{Z}_{\mathcal{H}}}{\mathcal{Z}_{n}}
$$

In this analysis, we will be interested in comparing the evidence for the precessing hypothesis $\mathcal{H}=p$ against the nonprecessing hypothesis $\mathcal{H}=n p$,

$$
\mathcal{B}_{p / n p}=\frac{\mathcal{Z}_{p}}{\mathcal{Z}_{n}} \frac{\mathcal{Z}_{n}}{\mathcal{Z}_{n p}}=\frac{\mathcal{Z}_{p}}{\mathcal{Z}_{n p}} .
$$

We perform Bayesian inference [133] on our simulated signals using the nested sampling algorithm [134-136] implemented in the publicly available inference library LALINFERENCE [137]. We inject the simulated signals into a zero-noise LIGO-Virgo three-detector network with a sensitivity representative of the first three months of the third observing run [138-140]. We marginalize over calibration uncertainties [141-143] using the representative values reported in [4] and start the likelihood integration at $20 \mathrm{~Hz}$. Our signal hypotheses will be two phenomenological waveform models IMRPhenomD (nonprecessing) [144,145] and IMRPhenomPv2 (precessing) [146]. We note that these two waveform models are not independent of each other; IMRPhenomPv2 is obtained by applying a rotation transformation to the quadrupolar modes of IMRPhenomD following the framework developed in Refs. [118,119,131]. The two phenomenological waveform models, however, differ in various aspects from our simulated signals; for example, they do not include higher-order modes and IMRPhenomPv2 uses fewer spin degrees of freedom to model precession. Hence systematic modeling errors due to inaccurate modeling or neglected physics are included in our analyses.

For the priors, we follow the choices as detailed in Appendix B of [4]. We use uniform priors on the component masses $m_{i} \in[1,40] M_{\odot}$, isotropic priors on the spin orientations, and a uniform prior on the dimensionless spin magnitudes $\chi_{i} \leqslant 0.99 .^{3}$ To enable a direct comparison to the precessing approximant, we use the $z$ prior for the spin priors (see, e.g., Appendix A of [147]) for IMRPhenomD. For the distance, we adopt a prior proportional to the luminosity distance squared with an upper cutoff of $600 \mathrm{Mpc}$.

From the one-dimensional posterior probability distribution function (PDF) we can obtain the parameter biases induced by systematics. Specifically, we define the bias as the difference between the maximum a posteriori (map) value of a parameter $x$ and its true value, i.e.,

$$
\Delta x:=x^{\text {map }}-x^{\text {true }} .
$$

For the effective precession spin parameter $\chi_{p}$ it follows that if $\Delta \chi_{p}>0$ the amount of precession in the system is overestimated and if $\Delta \chi_{p}<0$ it is underestimated.

Additionally, we also use the posterior quantile of the true parameter value $x^{\text {true }}$ given by

$$
Q(p):=\frac{1}{2}-\int_{x^{\min }}^{x^{\text {true }}} p(x \mid d, \mathcal{H}) \in[-0.5,0.5],
$$

as a measure of the displacement between the posterior median and the true value. For precession-related parameters $Q>0(Q<0)$ implies an overestimation (underestimation) of the amount of precession in the binary system. Moreover, the quantile also encodes the skew of the distribution.

To ascertain confidence in the measurement of precession, we additionally employ two statistical measures: the Kullback-Leibler divergence $D_{\mathrm{KL}}^{x}$ [148] and the (related) Jensen-Shannon divergence $D_{\mathrm{JS}}^{x}$ [149]. These two measures

\footnotetext{
${ }^{3}$ We note that this includes binaries with $\chi_{2}>0.7$, which is larger than the maximally allowed spin for neutron stars. However, we consider this choice appropriate due to the unknown nature of the secondary object.
} 

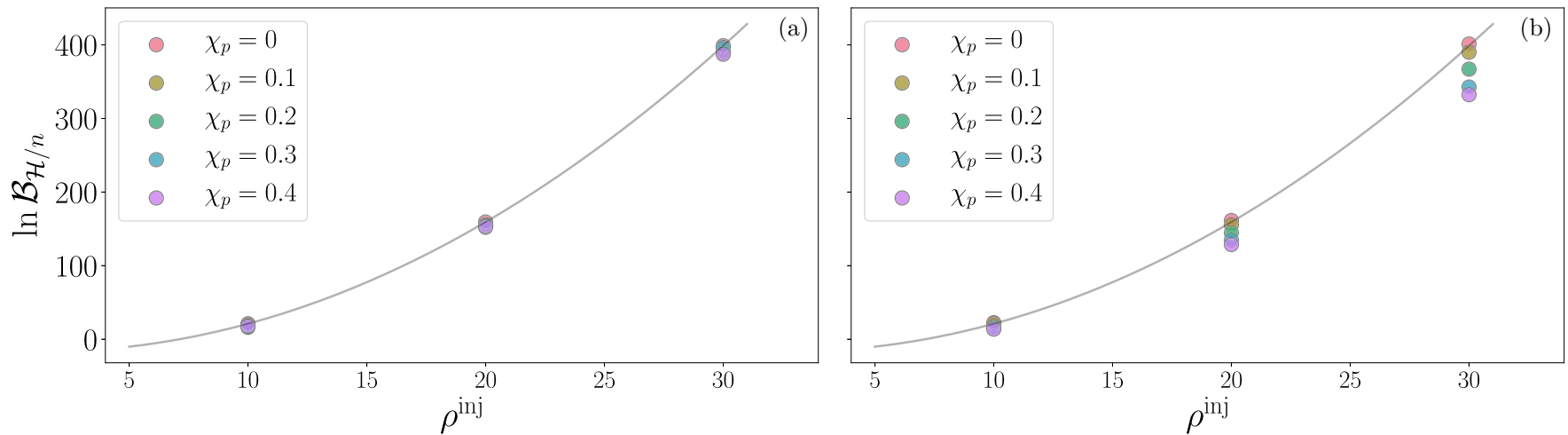

FIG. 1. Signal vs noise Bayes factor as a function of injected SNR $\rho^{\text {inj }}$ for (a) PhenomPv2 and (b) PhenomD for $q=9$ binaries for different values of $\chi_{p}$. The expected scaling $\ln \mathcal{B}_{\mathcal{H} / n} \propto\left(\rho^{\text {inj }}\right)^{2}$ (gray solid curve) is only recovered for (a) the precessing waveform model, with (b) significant deviations observed for the aligned-spin waveform model for $\chi_{p} \geqslant 0.2$ and $\rho>10$ due to the neglect of precession.

allow us to quantify the difference between two probability distribution $p(x)$ and $q(x)$ and are used to measure the information gain between the prior and the posterior distribution of a continuous random variable $x$. The Kullback-Leibler divergence is defined as

$$
D_{\mathrm{KL}}^{x}(p \mid q)=\int p(x) \log _{2}\left(\frac{p(x)}{q(x)}\right) d x .
$$

The Jensen-Shannon divergence, which defines a natural, normalized distance measure between two distributions, is given by

$$
D_{\mathrm{JS}}^{x}(p \mid q)=\frac{1}{2}\left[D_{\mathrm{KL}}^{x}(p \mid s)+D_{\mathrm{KL}}^{x}(q \mid s)\right],
$$

where $s=\frac{1}{2}(p+q)$.

\section{RESULTS}

\section{A. Precession measurements}

The Bayes factor between two hypotheses is a commonly used discriminator to assign confidence to a particular hypothesis (see, e.g., [150,151]). Here we treat the measurability of precession as a Bayesian model selection problem and use the Bayes factor between the precessing and the nonprecessing hypotheses to quantify the confidence to which we can measure precession. We first examine in detail a binary system with mass ratio $q=9$, consistent with the inferred mass ratio of GW190814 [2]. In particular, we investigate the measurability of precession as a function of injected SNR and precession spin $\chi_{p}$.

Figure 1 shows the signal versus noise Bayes factor (7) as a function of the injected signal SNR $\rho^{\text {inj }}$ for the precessing and the nonprecessing recovery models for different values of $\chi_{p}$. The Bayes factor for the signal-to-noise hypothesis approximately scales as $\ln \mathcal{B}_{\mathcal{H} / n} \propto\left(\rho^{\mathrm{inj}}\right)^{2}$ (see, e.g., [152]). For all values of $\chi_{p}$, we observe such a scaling when using the precessing waveform model. The nonprecessing model, however, shows significant deviations from this relation, especially for larger values of $\chi_{p}$ and with increasing SNR, where the nonprecessing waveform model systematically underestimates the injected SNR due to missing physics in the waveform approximant. In particular, we recall that neither recovery waveform model includes higher-order modes, while our simulated signals do. The results in Fig. 1 suggest, however, that higher-order modes play a subdominant role in comparison to precession (see also [153]). As a point of caution, we note that at high mass ratios and high $\chi_{p}$, IMRPhenomD shows strong systematic biases towards higher mass ratios. If we do not take appropriate care when choosing our priors, e.g., for $q$, this can lead to significant railing that impacts the calculation of the Bayes factors and the results can become unreliable.

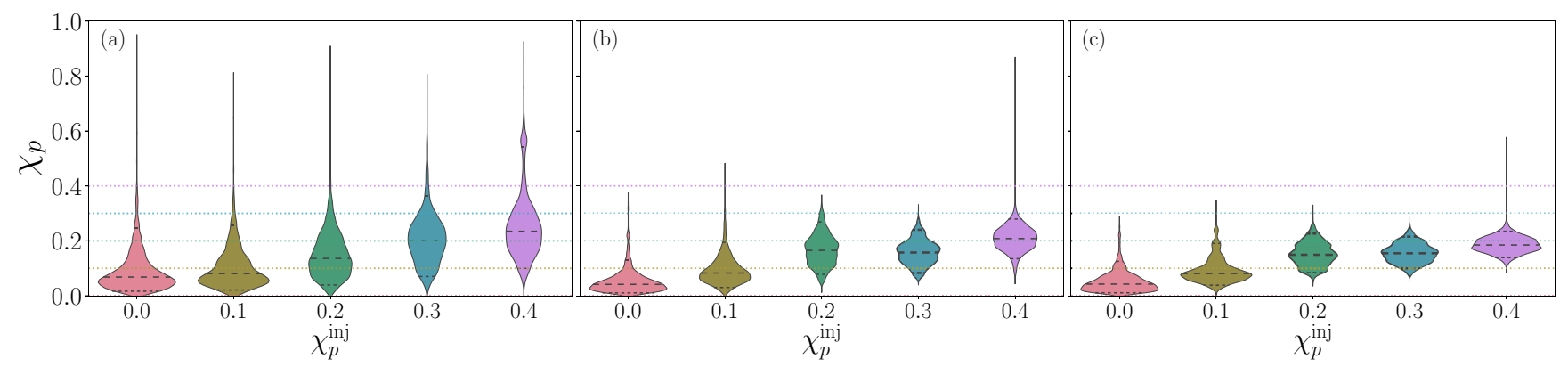

FIG. 2. One-dimensional PDF of the precession parameter $\chi_{p}$ for binaries with $q=9$ and varying amounts of precession (indicated on the $x$ axis) at three SNRs: (a) $\rho=10$, (b) $\rho=20$, and (c) $\rho=30$. The horizontal lines indicate the median and $90 \%$ CI. We see that the posterior width decreases as the SNR increases while the median changes only slightly, showing a clear systematic difference between the true $\chi_{p}$ value and the inferred median. 


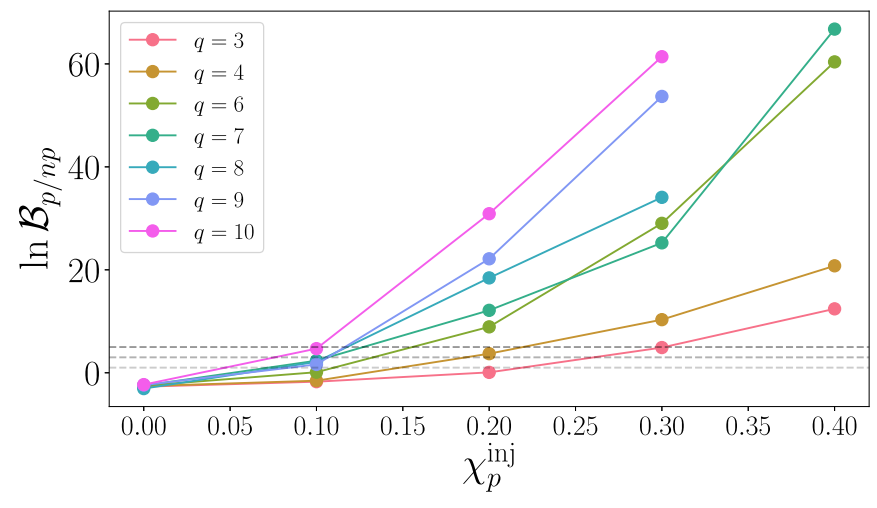

FIG. 3. Bayes factor for the precessing waveform model against the nonprecessing waveform model for varying mass ratio $q$ and the injected effective precession spin parameter $\chi_{p}$. Following Ref. [150], the horizontal dashed lines indicate positive (1.0), strong (3.0), and very strong (5.0) evidence against the nonprecessing hypothesis.

In Fig. 2 we show the one-dimensional posterior distributions for the effective spin parameter $\chi_{p}$ for the $q=9$ series at three different injected SNRs. We find that the precessing waveform model IMRPhenomPv2 systematically underestimates $\chi_{p}$ except for the nonprecessing case, i.e., $\chi_{p}=0$. We note, however, that this may be different for other binary inclinations.

As expected, with increasing SNR, tighter $90 \%$ credible interval $(\mathrm{CI})$ bounds are obtained and we find that the posterior widths scale proportional to $\rho^{-1}$, as anticipated in the highSNR limit [110,154]. The result in Fig. 2 also indicates that at higher SNRs, we can more confidently exclude the nonprecessing limit for smaller values of $\chi_{p}$ due to the reduction in posterior support as $\chi_{p} \rightarrow 0$. We note that the results discussed here correspond to injections in zero noise, as would be expected if averaged over many noise realizations. Individual noise realizations will induce a spread in the Bayes factors, though we leave a detailed characterization to further study.

We now turn to the analysis of the larger ensemble of mock GW signals described in Sec. III C. We recall that we have fixed the SNR to $\rho^{\text {inj }}=30$, which corresponds to moderately loud signals for current and near-future detector sensitivities [132]. Figure 3 shows the Bayes factor for the precessing versus the nonprecessing signal hypothesis (8) as a function of $\chi_{p}$ for all mass ratios considered. We find that for $q>$ 5 the precessing signal hypothesis is strongly favored (i.e., $\left.\ln \mathcal{B}_{p / n p} \geqslant 5\right)$ for $\chi_{p} \geqslant 0.2$. For lower mass ratios, a larger amount of precession, i.e., $\chi_{p}>0.3$, is required to clearly differentiate the nonprecessing from the precessing hypothesis. Similar to our observation for the $q=9$ case, we find that the Bayes factor becomes unreliable for high mass ratios and large amounts of precession; hence the data points for $\chi_{p}=0.4$ and $q \geqslant 8$ are omitted.

In addition to a Bayes factor in favor of precession, further evidence can be obtained directly from the inferred posterior distribution of $\chi_{p}$. We report two information gain measures $D_{\mathrm{JS}}^{\chi_{p}}$ and $D_{\mathrm{KL}}^{\chi_{p}}$, as defined in Eqs. (11) and (12). Due to the correlation between $\chi_{p}$ and $\chi_{\text {eff }}$, we condition the $\chi_{p}$ prior on the $\chi_{\text {eff }}$ posterior via rejection sampling following Ref. [4].

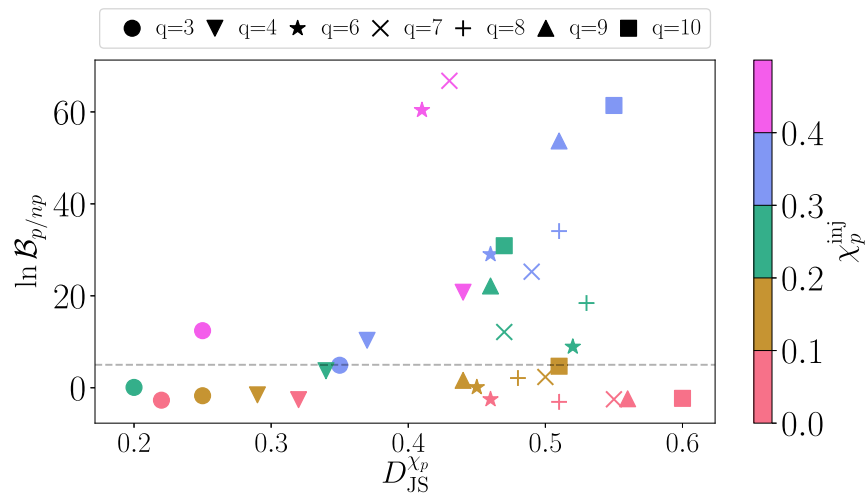

FIG. 4. Bayes factor vs the Jensen-Shannon divergence for all binaries considered here. The dashed horizontal line indicates a Bayes factor strongly favoring the precessing signal hypothesis.

These measures encapsulate how different the inferred posterior distribution of $\chi_{p}$ is in comparison to its prior distribution. Figure 4 shows the Jensen-Shannon divergence for $\chi_{p}$ vs the Bayes factor; the equivalent representation of $D_{\mathrm{KL}}^{\chi_{p}}$ can be found in Fig. 10 in the Appendix. The numerical values are reported in Table III also in the Appendix.

Focusing first on the normalized Jensen-Shannon divergence $D_{\mathrm{JS}}^{\chi_{p}} \in[0,1]$ (Fig. 4), we observe two general trends with minor fluctuations: (i) The divergence increases with mass ratio for all values of $\chi_{p}$ and (ii) for $q \geqslant 6, D_{\mathrm{JS}}$ decreases as $\chi_{p}$ increases. In all cases we find that information has been gained and for $q \geqslant 6$ the gain is greater than 0.4. Similar trends are observed for the KL divergence for all values of $\chi_{p}$. While these divergence measures are indicative of an appreciable difference between the prior and posterior distributions, on their own they are not enough to state whether or not precession has been identified. From Fig. 4, however, we notice clearly that nonprecessing or mildly precessing signals consistently disfavor the precessing hypothesis and have a large information gain that increases with the mass ratio.

This becomes further evident when contrasting the divergences with the median and $90 \% \mathrm{CI}$ of the $\chi_{p}$-posterior distributions given in Table II. For example, we consistently find a $D_{\mathrm{KL}}^{\chi_{p}} \gtrsim 1$ for the nonspinning and therefore nonprecessing binaries and, in combination with the median and $90 \%$ CI, we find that these binaries are correctly identified as nonprecessing or, at worst, as very mildly precessing. Furthermore, we find that the more asymmetric the mass ratio and the larger the intrinsic precession effects, the tighter and more confident the constraints that can be placed on $\chi_{p}$. This is also shown in Figs. 5(a) and 5(b) for binaries with mass ratios $q=3$ and 10, respectively; the results for the other mass ratios can be found in Fig. 11 in the Appendix. In particular, we find that a nonvanishing $\chi_{p}$ can be constrained away from zero with increasing significance as the mass ratio increases. For a true $\chi_{p} \geqslant 0.3$ we find that $\chi_{p}<0.08$ is excluded for all mass ratios at $99 \% \mathrm{CI}$; for mass ratios $q>4, \chi_{p}<0.07$ is excluded at $99 \%$ CI already for true $\chi_{p}$ values of 0.2 . This is not surprising as precession effects become more pronounced in this regime.

We notice, however, that while the divergence from the prior increases and the width of $90 \%$ CI shrinks with increas- 
TABLE II. Median and 90\% CI, bias, and quantile for $\chi_{p}$ for all mass ratios and injected $\chi_{p}$ values. We find that precession is clearly identified for $q \geqslant 6$ and $\chi_{p} \geqslant 0.2$ or lower mass ratio but higher precession spin. At the same time, when precession is inferred clearly, the amount of precession is consistently underestimated in comparison to the true injected value.

\begin{tabular}{|c|c|c|c|c|c|c|c|c|c|c|c|c|c|c|}
\hline \multirow[b]{2}{*}{$q$} & \multicolumn{5}{|c|}{$\chi_{p}$} & \multicolumn{5}{|c|}{$\Delta \chi_{p}$} & \multicolumn{4}{|c|}{$Q\left(\chi_{p}\right)$} \\
\hline & 0.0 & 0.1 & 0.2 & 0.3 & 0.4 & 0.0 & 0.1 & 0.2 & 0.3 & 0.4 & 0.1 & 0.2 & 0.3 & 0.4 \\
\hline 3 & $0.12_{-0.10}^{+0.29}$ & $0.12_{-0.08}^{+0.19}$ & $0.19_{-0.13}^{+0.22}$ & $0.24_{-0.10}^{+0.09}$ & $0.28_{-0.12}^{+0.21}$ & 0.21 & 0.09 & 0.03 & -0.14 & -0.17 & 0.1 & -0.03 & -0.36 & -0.33 \\
\hline 4 & $0.1_{-0.07}^{+0.16}$ & $0.12_{-0.08}^{+0.18}$ & $0.15_{-0.08}^{+0.13}$ & $0.22_{-0.08}^{+0.12}$ & $0.2_{-0.05}^{+0.12}$ & 0.09 & 0.01 & -0.07 & -0.10 & -0.19 & 0.1 & -0.26 & -0.39 & -0.5 \\
\hline 6 & $0.07_{-0.05}^{+0.09}$ & $0.09_{-0.05}^{+0.1}$ & $0.13_{-0.04}^{+0.07}$ & $0.19_{-0.07}^{+0.08}$ & $0.22_{-0.07}^{+0.11}$ & 0.03 & 0.06 & -0.07 & -0.15 & -0.20 & -0.09 & -0.44 & -0.49 & -0.5 \\
\hline 7 & $0.04_{-0.03}^{+0.09}$ & $0.09_{-0.05}^{+0.08}$ & $0.13_{-0.05}^{+0.08}$ & $0.18_{-0.07}^{+0.07}$ & $0.24_{-0.09}^{+0.07}$ & 0.09 & -0.01 & -0.09 & -0.15 & -0.17 & -0.07 & -0.42 & -0.49 & -0.5 \\
\hline 8 & $0.06_{-0.05}^{+0.08}$ & $0.09_{-0.04}^{+0.08}$ & $0.11_{-0.04}^{+0.07}$ & $0.17_{-0.05}^{+0.06}$ & $0.2_{-0.05}^{+0.05}$ & 0.10 & $<-10^{-3}$ & -0.03 & -0.11 & -0.2 & -0.18 & -0.48 & -0.5 & -0.5 \\
\hline 9 & $0.04_{-0.03}^{+0.08}$ & $0.08_{-0.04}^{+0.11}$ & $0.15_{-0.06}^{+0.08}$ & $0.15_{-0.06}^{+0.06}$ & $0.18_{-0.05}^{+0.05}$ & 0.02 & -0.01 & -0.02 & -0.17 & -0.18 & -0.19 & -0.36 & -0.5 & -0.5 \\
\hline 10 & $0.03_{-0.02}^{+0.06}$ & $0.08_{-0.03}^{+0.09}$ & $0.15_{-0.06}^{+0.07}$ & $0.16_{-0.05}^{+0.04}$ & $0.18_{-0.04}^{+0.05}$ & 0.02 & 0.01 & -0.03 & -0.12 & -0.23 & -0.22 & -0.38 & -0.5 & -0.5 \\
\hline
\end{tabular}

ing $\chi_{p}$, the recovery of $\chi_{p}$ is significantly biased (see the third column in Table II). For all mass ratios and values of $\chi_{p} \geqslant 0.2$, the amount of precession is consistently underestimated; only systems with low $\chi_{p}$ show small positive biases. Furthermore, for all configurations with $q \geqslant 6$ and $\chi_{p} \geqslant 0.2$ the true value of $\chi_{p}$ lies outside the $90 \%$ credible interval (see Fig. 11 in the Appendix), indicating that systematic modeling errors between SEOBNRv4PHM and IMRPhenomPv2 dominate over statistical uncertainty. Similarly, the posterior quantile

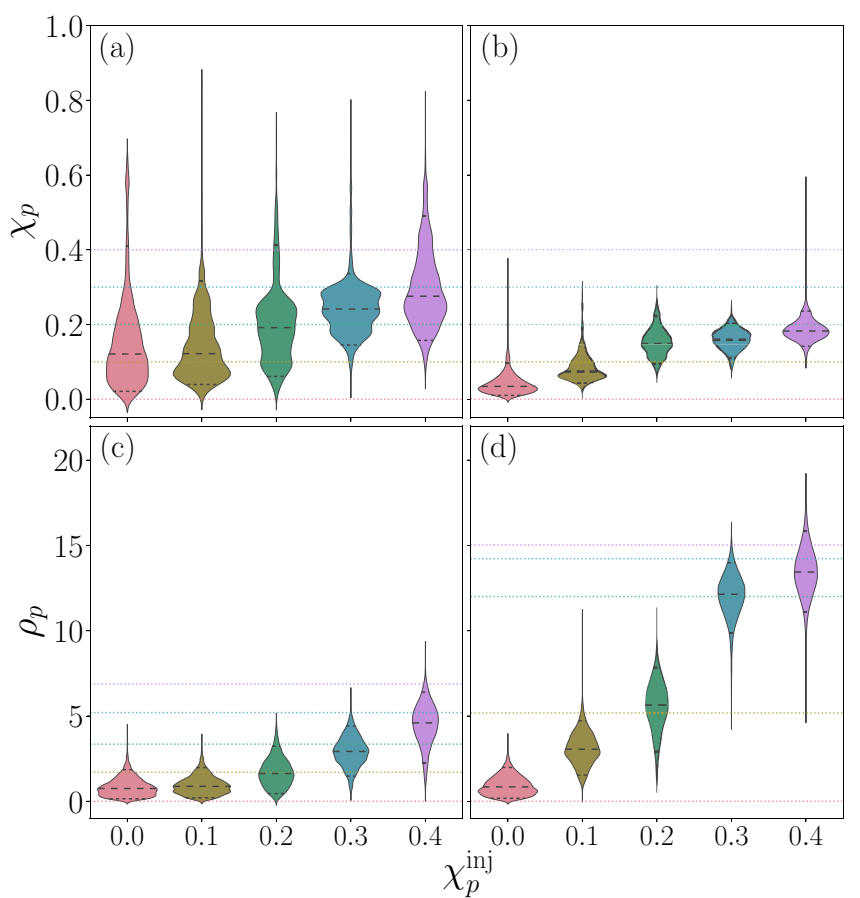

FIG. 5. Posterior distributions for $\chi_{p}$, (a) and (b), and $\rho_{p}$, (c) and (d), for mass ratios $q=3$, (a) and (c), and $q=10$, (b) and (d), for different amounts of precession (indicated on the $x$ axis). The black dashed and dotted lines within the shaded area indicate the median and 90\% CI, respectively. The colored dotted lines show the values of $\chi_{p}$ and $\rho_{p}$ for each injection.
$Q\left(\chi_{p}\right)$ as given by Eq. (10) reaffirms the appreciable underestimation of $\chi_{p}$ for $q \geqslant 4$ and $\chi_{p} \geqslant 0.2$ but additionally tells us about the skew of the inferred $\chi_{p}$ distribution. The observed biases in $\chi_{p}$ are perhaps not surprising given the differences between the injected waveform model and the one used for parameter recovery. Our results show that systematic modeling errors can affect the accuracy of spin measurements already at current detector sensitivities and relatively moderate SNRs. Consistent with the results obtained for GW190814, however, the absence of precession, i.e., $\chi_{p} \simeq 0$, is unlikely to be misidentified even for moderate inclinations. Our results indicate that precession (or the absence thereof) is robustly identified in such NSBH-like asymmetric binaries at reasonable SNRs and inclinations.

In addition to the fully Bayesian analysis, we now look at the distributions of the frequentist measure $\rho_{p}$ for all configurations (see Sec. III B). For each binary we compute the $\rho_{p}$ distribution from the posterior samples of the Bayesian analysis using PESUMMARY [155]. The distributions for $q=3$ and 10 are shown in Figs. 5(c) and 5(d); the results for the other mass ratios can be found in Fig. 11 in the Appendix.

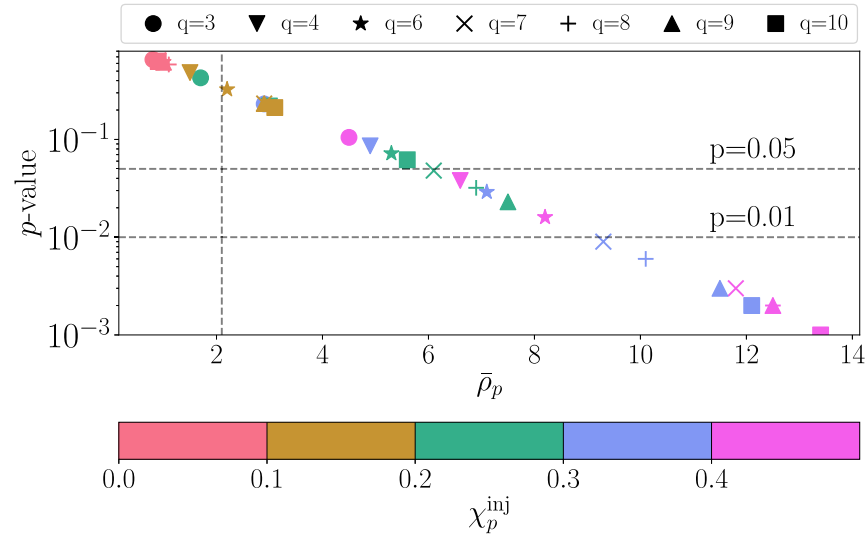

FIG. 6. One-sided $p$ value of $\rho_{p}$ as a function of the recovered mean precessing SNR $\bar{\rho}_{p}$. The dashed vertical line indicates $\rho_{p}=2.1$ and the dashed horizontal lines indicate $p$ values of 0.05 (moderate significance) and 0.01 (strong significance), respectively. 


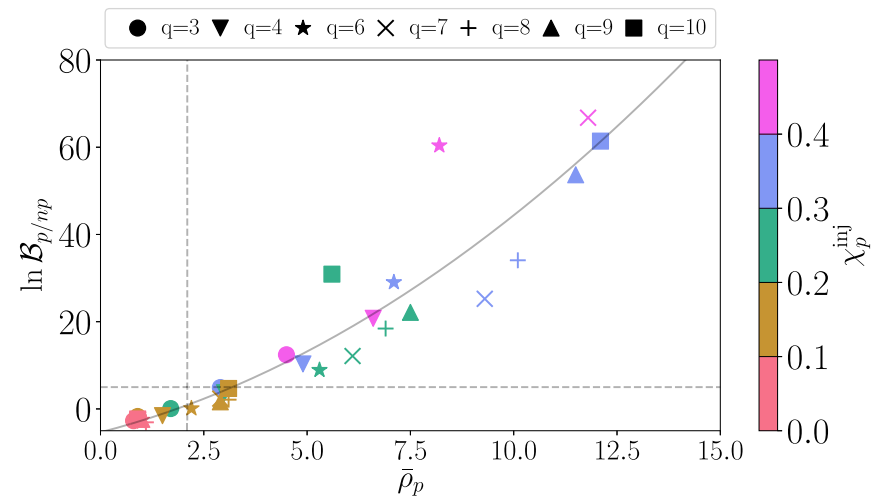

FIG. 7. Precessing vs nonprecessing Bayes factor for all binaries as a function of the mean recovered precessing SNR $\bar{\rho}_{p}$. The gray graph indicates the approximate quadratic dependence $\ln \mathcal{B}_{p / n p} \propto \bar{\rho}_{p}^{2}$.

The colored horizontal lines indicate the injected $\rho_{p}$ value for each value of $\chi_{p}$. We observe trends similar to $\chi_{p}$ : (i) The more asymmetric the mass ratio and the larger $\chi_{p}$ is, the more likely that $\rho_{p}$ exceeds the threshold of 2.1 ; (ii) $\rho_{p}$ is always underestimated except for the nonspinning cases, where it is overestimated.

To quantify the statistical significance of the inferred $\rho_{p}$, we compute the $p$ value for its mean relative to a $\chi^{2}$ distribution with two degrees of freedom, which is the distribution expected in the absence of precession [120]; the smaller the $p$ value, the more significant the deviation from the nonprecessing distribution. Figure 6 shows the $p$ value as a function of the recovered mean $\bar{\rho}_{p}$, where the two horizontal lines indicate $p$ values of 0.05 (moderate significance) and 0.01 (strong significance), respectively. The most significant $p$ values are obtained only for $\chi_{p} \geqslant 0.3$ and $q \geqslant 7$. We find the $\rho_{p}$ results to be consistent with the results from the fully Bayesian analysis, but they do not provide any additional information or further constraining power. In particular, the $p$-value statistic suggests that the two-harmonic threshold precessing SNR of 2.1 is too low in the presence of systematic errors. The means, $1 \sigma$ variances, and $p$ values are given in Table IV in the Appendix.

In Fig. 7 we also show the precessing vs nonprecessing Bayes factor as a function of the recovered precessing SNR $\bar{\rho}_{p}$. We find that $\ln \mathcal{B}_{p / n p} \propto \bar{\rho}_{p}^{2}$. Similar to the $p$-value results, we see that confident statements about the presence of precession are restricted to larger mass ratios and in-plane spin values with a $\bar{\rho}_{p}$ value of 2.1 being too low a detection threshold in the presence of waveform systematics. By mapping the recovered precessing SNR to the Bayes factors, it will be possible to estimate the measurability of precession while avoiding additional parameter estimation runs. However, this requires a detailed characterization of the mapping and the impact of waveform systematics.

\section{B. Mass-spin degeneracy}

Accurate measurement of the component masses is of vital importance in determining the astrophysical nature of low-mass compact objects. This is particularly important for NSBH-like binaries, where there is likely to be no EM counterpart and no discernible information regarding the tidal deformability of the lighter companion [23]. In this section, we assess the confidence to which we can measure the secondary mass in high-mass-ratio binaries. We focus on two
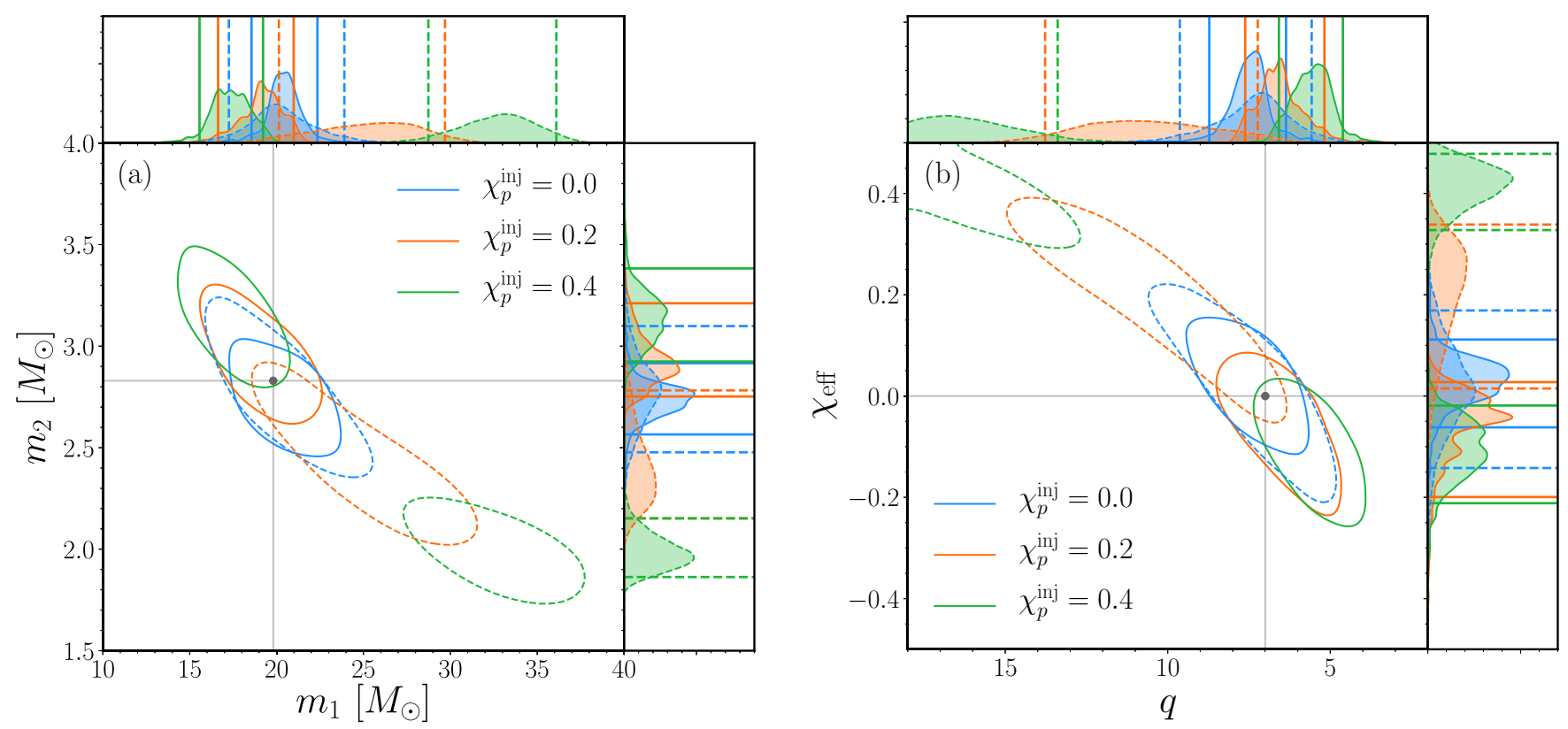

FIG. 8. One-dimensional and joint posterior distributions for (a) the component masses and (b) the mass ratio and effective aligned spin, for a set of $q=7$ binaries as inferred using the IMRPhenomPv2 (solid lines) and IMRPhenomD (dashed lines) waveform models. As we increase the amount of precession in the injected signal, we find a significant increase in the bias of the inferred masses towards smaller values when recovering with the nonprecessing approximant. As can be seen, spin precession breaks the mass-spin degeneracy in the $q-\chi_{\text {eff }}$ plane [44], allowing a tighter localization of both the mass and the spin. 

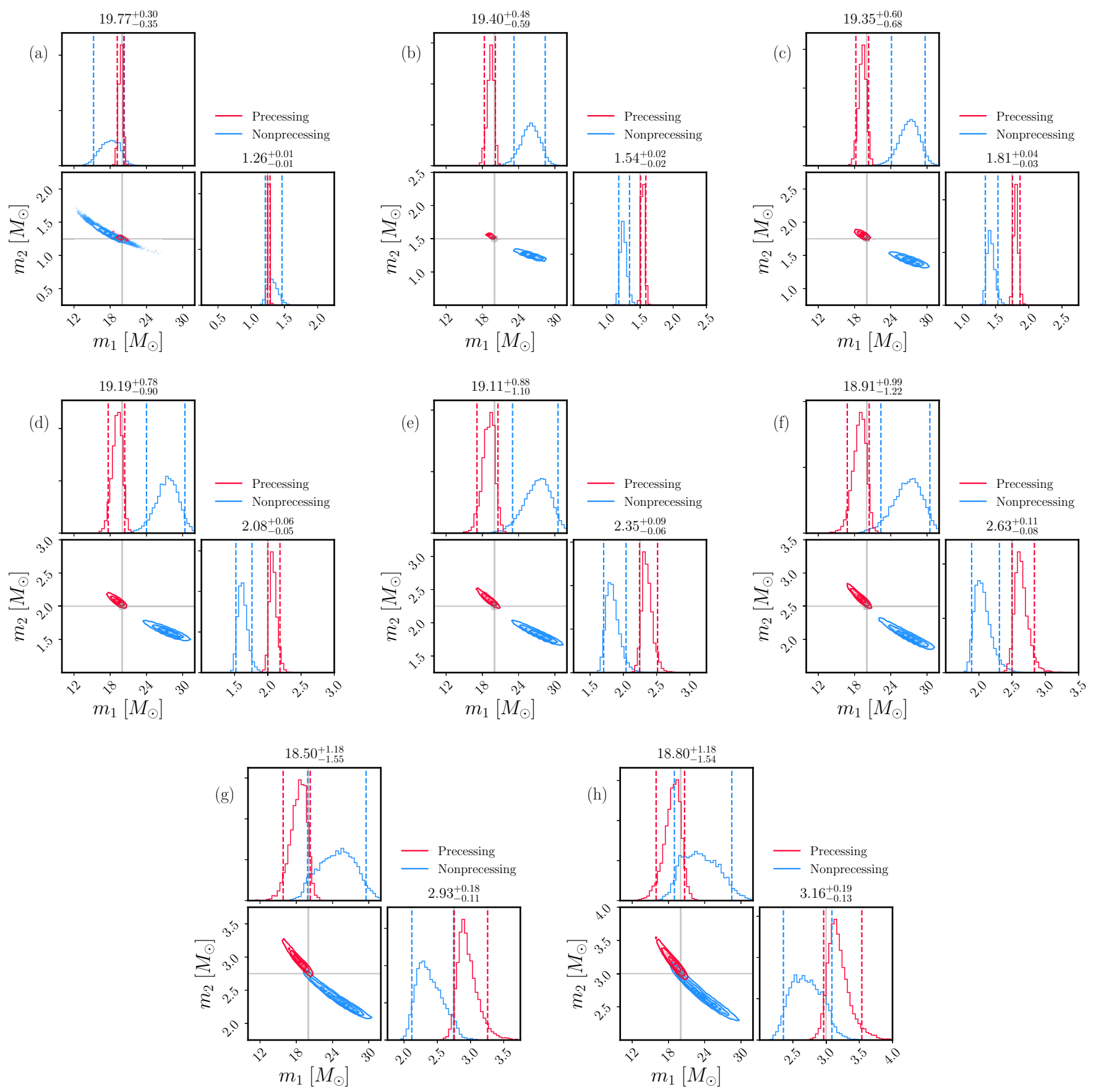

FIG. 9. One-dimensional and joint mass posteriors for the NSBH-like series of injections. We keep the mass of the primary fixed at $m_{1}=20 M_{\odot}$ and vary the mass of the secondary in the range $m_{2} \in[1.25,3.00] M_{\odot}$. The SNR of the binary is fixed at $\rho=30$ with $\chi_{\text {eff }}=0$ and $\chi_{p}=0.2$. Spin precession breaks the mass-spin degeneracy, leading to significant improvements in the recovered mass parameters. The nonprecessing templates (blue lines) demonstrate a systematic bias towards heavier primary and lighter secondary masses compared to the precessing templates (red lines). The gray lines denote the injected component masses. The values reported are the $90 \%$ from the precessing model IMRPhenomPv2 .

scenarios. In the first scenario, we highlight how the biases in the inferred component masses become progressively worse as we increase the amount of precession in the system. In the second scenario, we consider an astrophysically motivated series in which we fix the mass of the primary and vary the mass of the secondary such that it spans a range of plausible neutron-star masses $[6,17,156]$.
In Fig. 8 we show the one-dimensional and joint posteriors for the source frame component masses [Fig. 8(a)] for a $q=$ 7 binary with $\mathcal{M}_{c}=6 M_{\odot}, \chi_{p} \in\{0,0.2,0.4\}$, and all other extrinsic parameters fixed to the values reported in Table I. We show both the precessing (solid lines) and nonprecessing (dashed lines) posteriors. By neglecting spin precession in the recovery waveform model, we find significant biases in the inferred component masses as the magnitude of the in-plane 
spin is increased. For the most strongly precessing configurations considered here, $\chi_{p}=0.4$, the bias in the primary mass is $\Delta m_{1} \simeq+13 M_{\odot}$, and in the secondary $\Delta m_{2} \simeq-0.88 M_{\odot}$, respectively. In particular, this example demonstrates how a compact object with mass $m_{2}=2.83 M_{\odot}$, which is significantly heavier than the most massive NS observed to date [15], would be misidentified as having a mass of $\simeq 1.95 M_{\odot}$ if spinprecession effects were neglected. Similarly, in Fig. 8(b), we highlight how spin-precession breaks the $q$ - $\chi_{\text {eff }}$ degeneracy [44,45,47].

For the second series, the mass of the primary is fixed to $m_{1}=20 M_{\odot}$ and the secondary mass varies from $m_{2}=$ $1.25 M_{\odot}$ to $m_{2}=3.00 M_{\odot}$. Here we allow for a small but nonnegligible amount of precession with $\chi_{p}=0.2$. The results are shown in Fig. 9. For all binaries considered in this series, the posteriors obtained using IMRPhenomPv2 are demonstrably less biased, with the true injected masses being always contained within the $90 \%$ CI. In addition, the posteriors are tighter than the posteriors inferred using IMRPhenomD. As we increase $m_{2}$, we increase $\mathcal{M}_{c}$ but decrease the mass ratio. Consequentially, we find that the IMRPhenomD posteriors become progressively less biased but the posteriors widths become broader. We observe that the nonprecessing approximant significantly underestimates the mass of the secondary for nearly all binaries considered, leading to stronger support for masses that are consistent with known theoretical bounds on the maximum NS mass. In contrast, as IMRPhenomPv2 is recovering almost unbiased mass estimates, with the true injected value always lying towards the lower $90 \% \mathrm{CI}$, the posterior support for plausible neutron-star masses is significantly reduced. Of particular note is the $m_{2}=2.75 M_{\odot}$ injection, falling just above current causal bounds on the NS mass, where IMRPhenomPv2 demonstrates little posterior support for $m_{2}<2.75 M_{\odot}$ whereas IMRPhenomD has posterior support down to $2 M_{\odot}$; also see Fig. 12 for a comparison of the inferred one-dimensional posterior distributions for $m_{2}$. While only a preliminary study on a single set of injections, these results serve to highlight the importance of including spin precession in our waveform models when making inferences about the nature of the secondary compact object $[67,77]$. We note that misidentifying a light $\mathrm{BH}$ as a heavy NS will introduce significantly less bias in inferred NS parameters than misidentifying a $\mathrm{BH}$ as a light NS [67]. In such scenarios, the use of nonprecessing approximants for parameter estimation could introduce nontrivial biases in the inferred population properties, including inferences on the NS equation of state $[14,16,157]$.

As a caveat to the analysis discussed here, we neglect the role that tidal effects and tidal disruption could have on the morphology of a NSBH waveform [22,34,36-41]. As we move to larger mass ratios, the occurrence of tidal disruption becomes increasingly unlikely and the waveform begins to closely resemble that of a $\mathrm{BBH}$ with the high-frequency behavior of the amplitude being governed by the ringdown of the primary $\mathrm{BH}$ [158]. For more comparable mass ratios, significant tidal disruption of the NS can take place and the amplitude becomes exponentially suppressed at high frequencies. Several nonprecessing waveform models have incorporated such effects [159-162] but no precessing NSBH waveform models are yet available. The impact of tidal disruption on statistical and systematic uncertainties in nonspinning NSBH binaries has recently been investigated in [163], where it was shown that neglecting tidal contributions introduces systematic biases for comparable mass ratios, but at these highly asymmetric mass ratios spin effects are expected to be the more important one.

\section{DISCUSSION}

Accurate measurements of the component masses and misaligned spins are of prime importance in understanding the origin and evolution of astrophysical compact binaries. It is therefore imperative that we understand how robust such measurements from GW observations are. In this work, we have reassessed our ability to discern spin precession in high-massratio binaries similar to GW190814 in the current detector era. We have quantified this using Bayesian model selection supplemented by additional Bayesian and frequentist measures.

Due to the large number of parameters that characterize a precessing compact binary, many studies on the measurability of precession have commonly focused on statements made at the population level $[48,59,65]$. Detailed systematic studies are rare [153,164-166]. Here we consider a restricted series of injections designed to understand how systematically increasing the amount of precession impacts our ability to make statements on the measurability of precession in GW190814-

TABLE III. Information gain (in bits) between the prior and posterior for $\chi_{p}$. We show the $D_{\mathrm{JS}}$ and $D_{\mathrm{KL}}$ divergences at all mass ratios and spins considered. We condition the prior on $\chi_{p}$ by the posteriors on $\chi_{\text {eff }}$.

\begin{tabular}{|c|c|c|c|c|c|c|c|c|c|c|}
\hline \multirow[b]{2}{*}{$q$} & \multicolumn{5}{|c|}{$D_{\mathrm{JS}}^{\chi_{p}}\left(\chi_{\mathrm{eff}}\right)(\mathrm{bits})$} & \multicolumn{5}{|c|}{$D_{\mathrm{KL}}^{\chi_{p}}\left(\chi_{\mathrm{eff}}\right)(\mathrm{bits})$} \\
\hline & 0.0 & 0.1 & 0.2 & 0.3 & 0.4 & 0.0 & 0.1 & 0.2 & 0.3 & 0.4 \\
\hline 3 & 0.22 & 0.25 & 0.20 & 0.35 & 0.25 & 0.92 & 0.90 & 0.68 & 1.35 & 0.83 \\
\hline 4 & 0.32 & 0.29 & 0.34 & 0.37 & 0.44 & 1.29 & 1.23 & 1.19 & 1.32 & 1.70 \\
\hline 6 & 0.46 & 0.45 & 0.52 & 0.46 & 0.41 & 1.75 & 1.85 & 2.20 & 1.58 & 1.45 \\
\hline 7 & 0.55 & 0.50 & 0.47 & 0.49 & 0.43 & 2.30 & 1.85 & 1.81 & 1.80 & 1.68 \\
\hline 8 & 0.51 & 0.48 & 0.53 & 0.51 & 0.55 & 2.05 & 1.93 & 1.95 & 1.91 & 2.23 \\
\hline 9 & 0.56 & 0.44 & 0.46 & 0.51 & 0.56 & 2.39 & 1.73 & 1.73 & 1.90 & 2.29 \\
\hline 10 & 0.60 & 0.51 & 0.47 & 0.55 & 0.56 & 2.59 & 2.07 & 1.82 & 2.21 & 2.23 \\
\hline
\end{tabular}




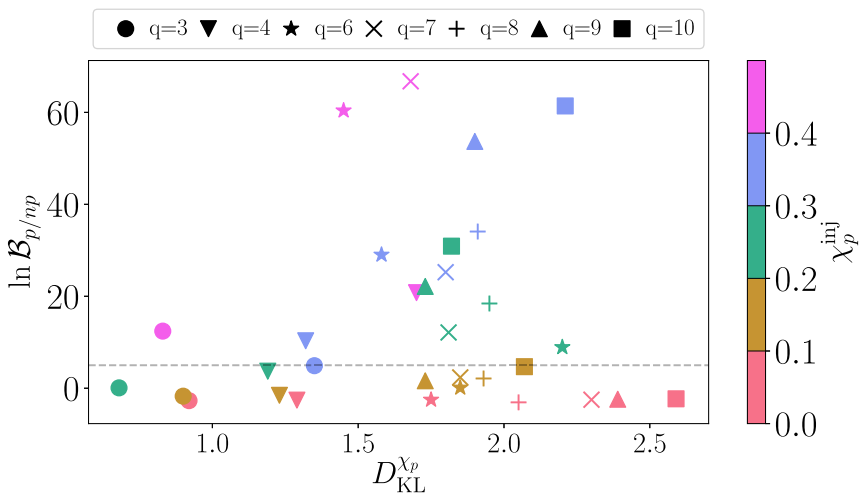

FIG. 10. Bayes factor vs the KL divergence for all binaries considered here. The dashed horizontal line indicates a Bayes factor of 5 , strongly favoring the precessing signal hypothesis.

like systems and how the neglect of precession in waveform models leads to nontrivial biases in the inferred component masses, which can have crucial implication for NSBH-like systems.

Our results show that even small amounts of precession are robustly identified for moderately asymmetric mass ratios $q>$ 5. For less unequal masses, larger amounts of precession are required to make robust statements. For all mass ratios we find that model selection alone does not allow us to differentiate between a nonprecessing binary and a binary with $\chi_{p}<0.1$; for small asymmetric mass ratios an even larger amount of precession is required for model selection to discriminate. For all mass ratios $q>3$ precession with $\chi_{p}>0.2$ is robustly measured but biased towards lower values, showing that sys- tematic errors can already be of concern at current detector sensitivities. As illustrated for the $q=9$ case, we expect that lower SNR signals will need to be more strongly precessing to obtain a Bayes factor high enough to distinguish between the precessing and nonprecessing hypotheses. For binaries with higher chirp masses, where fewer precession cycles are detectable, preliminary studies show similar trends, but we leave a comprehensive analysis to future work. As for smaller (larger) inclinations, previous work suggests that it will be more (less) difficult to identify precession conclusively [167].

Furthermore, our analysis highlights how even relatively mild amounts of precession can lead to significant biases in the inferred component masses. Systematically increasing the amount of precession in the system leads to a significant overestimation (underestimation) of the primary (secondary) mass when using an aligned-spin approximant. Precession also breaks the mass-spin degeneracy, and we consequently find that the posterior widths for the component masses inferred using a nonprecessing approximant are a factor $\sim 2$ broader than the equivalent posteriors inferred using the precessing approximant.

In our analyses, we used a fixed inclination and polarization and systematically varied the mass ratio $q$ and spin precession $\chi_{p}$. We restricted our analysis to NSBH-like binaries whose chirp mass is consistent with the values reported for GW190814 [2]. Larger studies exploring the full dependence on the sky location, orientation, masses, and full spin degrees of freedom will be important but are beyond the scope of this paper. Further, we only consider binaries with $\chi_{\text {eff }} \sim 0$, which is consistent with current observations [4] and theoretical modeling of NSBH systems which predicts large spin misalignment for a high fraction of binaries [50].

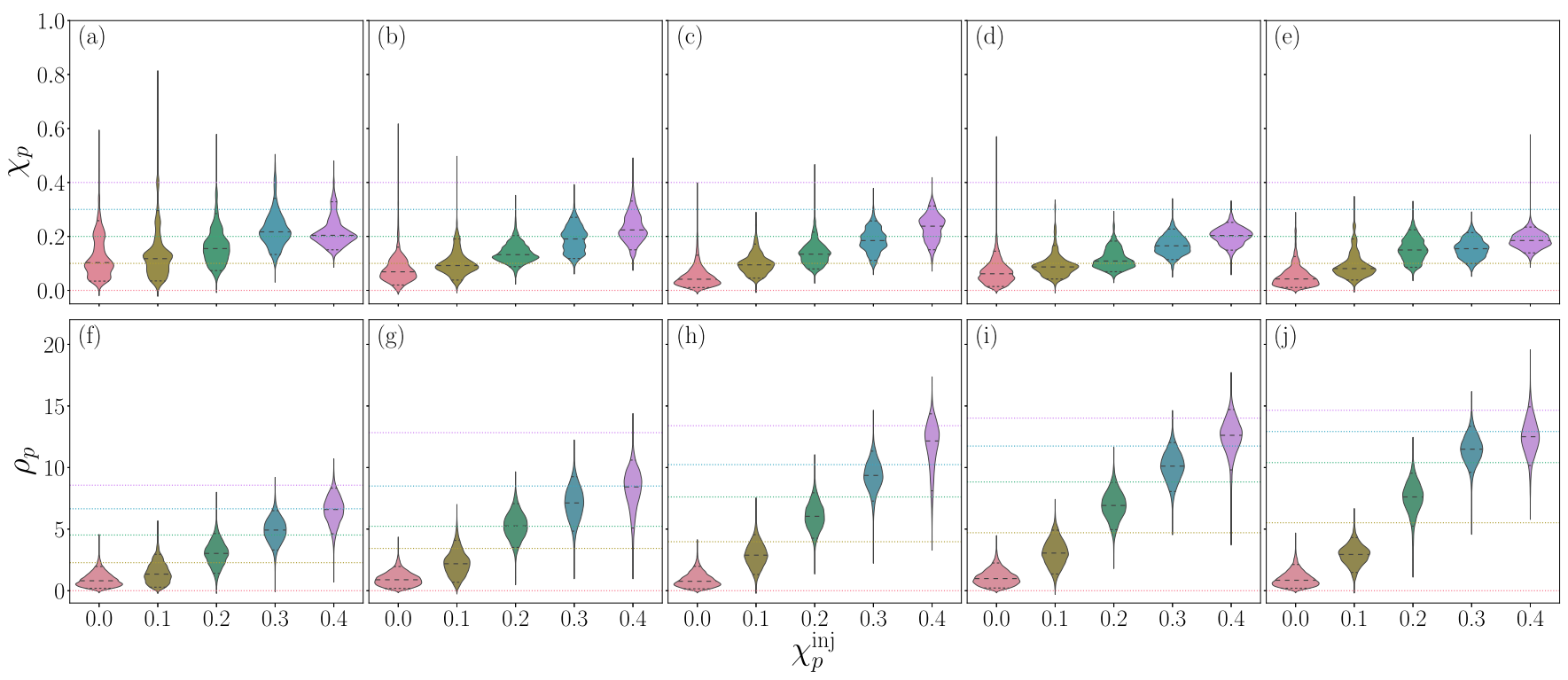

FIG. 11. One-dimensional posterior distributions for (a)-(e) $\chi_{p}$ and (f)-(j) $\rho_{p}$ for mass ratios (a) $q=4$, (b) $q=6$, (c) $q=7$, (d) $q=8$, and (e) $q=9$ as a function of increasing $\chi_{p}$ indicated on the $x$ axis. The lines within the shaded area indicate the median (dashed) and $90 \%$ CI (dotted). The colored dotted lines show the value of $\chi_{p}$ and $\rho_{p}$ for each injection. Here $\chi_{p}$ is constrained away from zero with increasing significance as the mass ratio and injected $\chi_{p}$ increase. For all configurations with $q \geqslant 6$ and $\chi_{p} \geqslant 0.2$ the true value of $\chi_{p}$ lies outside the $90 \%$ credible interval, showing that systematic errors start to exceed the statistical uncertainty. The $\rho_{p}$ distributions show the same trends, with $\rho_{p}$ only exceeding the threshold of 2.1 at the $1 \sigma$ level for injections with $q \geqslant 6$ and $\chi_{p} \geqslant 0.2$. 
TABLE IV. Mean and $1 \sigma$ variance of $\rho_{p}$ and its $p$ value for all binary configurations. The $p$ value is calculated for the mean with respect to a $\chi^{2}$ distribution with two degrees of freedom, which corresponds to the nonprecessing case. The lower the $p$ value, the more significant the deviation from the nonprecessing $\chi^{2}$ distribution.

\begin{tabular}{|c|c|c|c|c|c|c|c|c|c|c|}
\hline \multirow[b]{2}{*}{$q$} & \multicolumn{5}{|c|}{$\bar{\rho}_{p}$} & \multicolumn{5}{|c|}{$p$ value } \\
\hline & 0.0 & 0.1 & 0.2 & 0.3 & 0.4 & 0.0 & 0.1 & 0.2 & 0.3 & 0.4 \\
\hline 3 & $0.8 \pm 0.3$ & $0.9 \pm 0.3$ & $1.7 \pm 0.7$ & $2.9 \pm 0.8$ & $4.5 \pm 1.5$ & 0.657 & 0.622 & 0.427 & 0.231 & 0.105 \\
\hline 4 & $0.9 \pm 0.3$ & $1.5 \pm 0.7$ & $3.0 \pm 1.0$ & $4.9 \pm 1.0$ & $6.6 \pm 1.3$ & 0.634 & 0.483 & 0.220 & 0.086 & 0.038 \\
\hline 6 & $1.0 \pm 0.3$ & $2.2 \pm 1.1$ & $5.3 \pm 1.2$ & $7.1 \pm 1.9$ & $8.2 \pm 2.8$ & 0.620 & 0.325 & 0.072 & 0.029 & 0.016 \\
\hline 7 & $0.9 \pm 0.3$ & $2.9 \pm 1.0$ & $6.1 \pm 1.3$ & $9.3 \pm 1.5$ & $11.8 \pm 3.5$ & 0.644 & 0.233 & 0.048 & 0.009 & 0.003 \\
\hline 8 & $1.1 \pm 0.4$ & $3.1 \pm 1.1$ & $6.9 \pm 1.4$ & $10.1 \pm 1.5$ & $12.5 \pm 2.3$ & 0.585 & 0.214 & 0.032 & 0.006 & 0.002 \\
\hline 9 & $1.0 \pm 0.4$ & $2.9 \pm 0.7$ & $7.5 \pm 1.8$ & $11.5 \pm 1.3$ & $12.5 \pm 2.1$ & 0.616 & 0.233 & 0.023 & 0.003 & 0.002 \\
\hline 10 & $0.9 \pm 0.3$ & $3.1 \pm 0.9$ & $5.6 \pm 2.2$ & $12.1 \pm 1.6$ & $13.4 \pm 2.2$ & 0.627 & 0.212 & 0.062 & 0.002 & 0.001 \\
\hline
\end{tabular}

The analyses presented in this paper could be improved by incorporating higher modes [49,168-171] and improved modeling of precession $[122,172,173]$ into the recovery waveform, where we anticipate tighter constraints on the component masses, spins, and the orientation of the binary. Further, for lower-mass-ratio binaries $(q \leqslant 4)$, tidal effects which are not included in our analysis may become important. Since tidal parameters are also correlated with the mass, waveforms that include finite-size effects, tidal disruption, and precession will be relevant [67].

The detection of GW190412 and GW190814 provided the first GW observations of highly asymmetric compact binaries. This has created an opportunity for novel relativistic effects, including spin precession and higher-order modes. As gravitational-wave detectors approach design sensitivity, it will be increasingly important to understand systematic errors in the waveform models and the impact on parameter estimation.

\section{ACKNOWLEDGMENTS}

We thank Alberto Vecchio and Serguei Ossokine for useful discussions, Richard O'Shaughnessy for comments on the manuscript, and Stephen Fairhurst, Rhys Green, Mark Hannam, and Charlie Hoy for providing early access to the code used to calculate $\rho_{p}$ We are grateful for computational resources provided by Cardiff University and funded by STFC Grants No. ST/I006285/1 and No. ST/V001167/1 supporting the UK Involvement in the Operation of Advanced LIGO. P.S. acknowledges NWO Veni Grant No. 680-47-460. R.B. was supported by the School of Physics and Astronomy at the University of Birmingham and the Birmingham Institute for Gravitational Wave Astronomy. L.M.T. was supported by STFC, the School of Physics and Astronomy at the University of Birmingham, and the Birmingham Institute for Gravitational Wave Astronomy. This paper has the LIGO document No. P2000224.
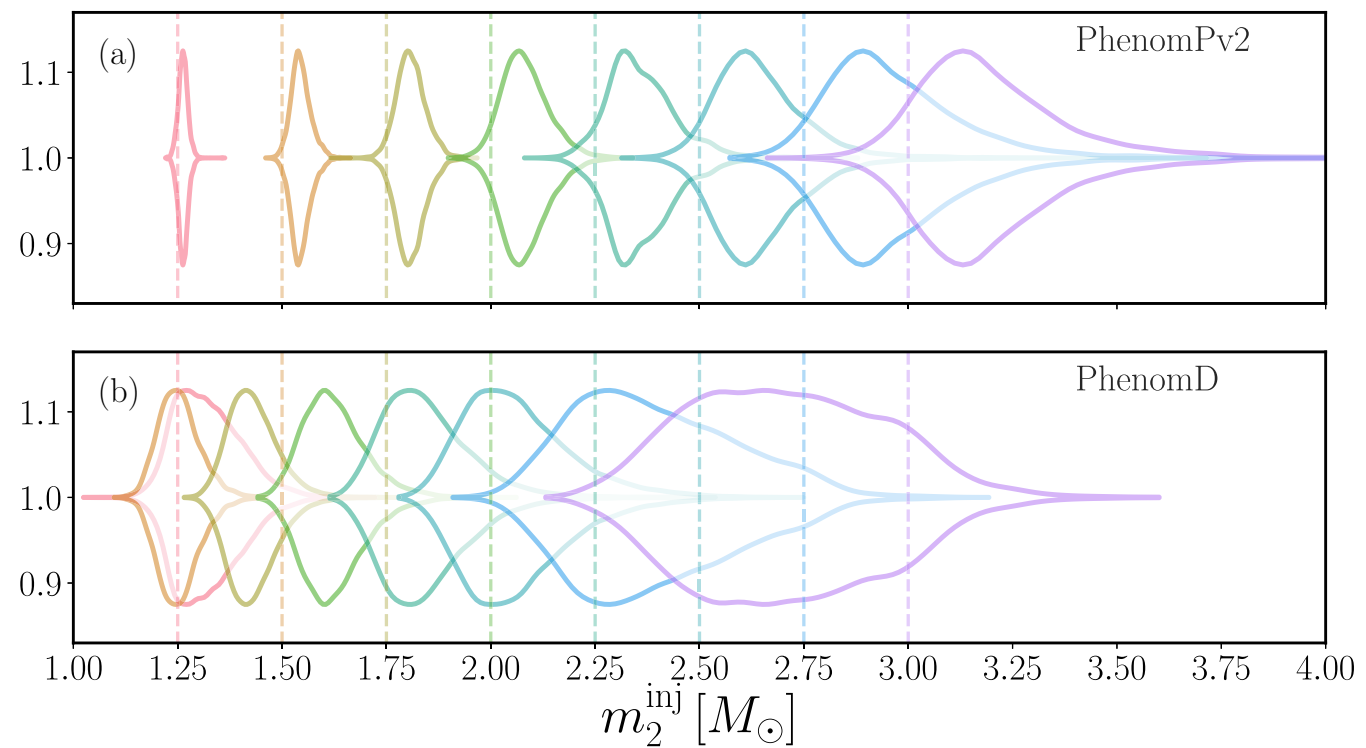

FIG. 12. One-dimensional posterior distributions for secondary mass as a function of the injected secondary mass (vertical dashed lines). Posteriors were obtained using (a) IMRPhenomPv2 and (b) IMRPhenomD. As highlighted in Sec. IV B, spin-precession breaks the mass-spin degeneracy allowing for tighter constraints on the component masses. For all injections considered here, IMRPhenomD both systematically underestimates the mass of the secondary and has significantly broader posteriors. 


\section{APPENDIX: SUPPLEMENTARY INFORMATION}

In addition to the figures and tables in the main text, we provide further details and complementary figures here.

Table III gives the numerical values for the JensenShannon and the Kullback-Leibler (KL) divergences for $\chi_{p}$. Figure 10 is the equivalent of Fig. 4 for the KL divergence.
Figure 11 is the complement to Fig. 5 in Sec. IV A showing the results for the remaining mass ratios as detailed in Sec. III C.

In Table IV we give the numerical values obtained for the mean precessing SNR $\bar{\rho}_{p}$ and the corresponding $p$ values.

In Fig. 12 we show the one-dimensional posterior distributions of the secondary mass as a function of the injected value.
[1] R. Abbott et al. (LIGO Scientific Collaboration and Virgo Collaboration), Phys. Rev. D 102, 043015 (2020).

[2] R. Abbott et al. (LIGO Scientific Collaboration and Virgo Collaboration), Astrophys. J. 896, L44 (2020).

[3] B. Abbott et al. (LIGO Scientific Collaboration and Virgo Collaboration), Phys. Rev. X 6, 041015 (2016); 8, 039903(E) (2018).

[4] B. Abbott et al. (LIGO Scientific Collaboration and Virgo Collaboration), Phys. Rev. X 9, 031040 (2019).

[5] C. D. Bailyn, R. K. Jain, P. Coppi, and J. A. Orosz, Astrophys. J. 499, 367 (1998).

[6] F. Özel, D. Psaltis, R. Narayan, and J. E. McClintock, Astrophys. J. 725, 1918 (2010).

[7] W. M. Farr, N. Sravan, A. Cantrell, L. Kreidberg, C. D. Bailyn, I. Mandel, and V. Kalogera, Astrophys. J. 741, 103 (2011).

[8] F. Özel, D. Psaltis, R. Narayan, and A. S. Villarreal, Astrophys. J. 757, 55 (2012).

[9] L. Kreidberg, C. D. Bailyn, W. M. Farr, and V. Kalogera, Astrophys. J. 757, 36 (2012).

[10] P. C. C. Freire, S. M. Ransom, S. Begin, I. H. Stairs, J. W. Hessels, L. H. Frey, and F. Camilo, Astrophys. J. 675, 670 (2008).

[11] J. M. Corral-Santana, J. Casares, T. Munoz-Darias, F. E. Bauer, I. G. Martinez-Pais, and D. M. Russell, Astron. Astrophys. 587, A61 (2016).

[12] J. Alsing, H. O. Silva, and E. Berti, Mon. Not. R. Astron. Soc. 478, 1377 (2018).

[13] M. Shibata, S. Fujibayashi, K. Hotokezaka, K. Kiuchi, K. Kyutoku, Y. Sekiguchi, and M. Tanaka, Phys. Rev. D 96, 123012 (2017).

[14] B. P. Abbott et al. (LIGO Scientific Collaboration and Virgo Collaboration), Class. Quantum Grav. 37, 045006 (2020).

[15] H. T. Cromartie et al., Nat. Astron. 4, 72 (2019).

[16] R. Essick, P. Landry, and D. E. Holz, Phys. Rev. D 101, 063007 (2020).

[17] B. Abbott et al. (LIGO Scientific Collaboration and Virgo Collaboration), Astrophys. J. Lett. 892, L3 (2020).

[18] L.-X. Li and B. Paczynski, Astrophys. J. Lett. 507, L59 (1998).

[19] S. Rosswog, Astrophys. J. 634, 1202 (2005).

[20] M. Shibata and K. Taniguchi, Phys. Rev. D 73, 064027 (2006).

[21] B. Metzger, G. Martinez-Pinedo, S. Darbha, E. Quataert, A. Arcones, D. Kasen, R. Thomas, P. Nugent, I. Panov, and N. Zinner, Mon. Not. R. Astron. Soc. 406, 2650 (2010).

[22] F. Foucart, Phys. Rev. D 86, 124007 (2012).

[23] F. Pannarale and F. Ohme, Astrophys. J. Lett. 791, L7 (2014).

[24] I. Arcavi et al., Nature (London) 551, 64 (2017).

[25] B. Abbott et al. (LIGO Scientific Collaboration and Virgo Collaboration, Fermi GBM, INTEGRAL, IceCube, AstroSat
Cadmium Zinc Telluride Imager Team, IPN, Insight-Hxmt, ANTARES, Swift, AGILE Team, 1M2H Team, Dark Energy Camera GW-EM, DES, DLT40, GRAWITA, Fermi-LAT, ATCA, ASKAP, Las Cumbres Observatory Group, OzGrav, DWF (Deeper Wider Faster Program), AST3, CAASTRO, VINROUGE, MASTER, J-GEM, GROWTH, JAGWAR, CaltechNRAO, TTU-NRAO, NuSTAR, Pan-STARRS, MAXI Team, TZAC Consortium, KU, Nordic Optical Telescope, ePESSTO, GROND, Texas Tech University, SALT Group, TOROS, BOOTES, MWA, CALET, IKI-GW Follow-up, H.E.S.S., LOFAR, LWA, HAWC, Pierre Auger, ALMA, Euro VLBI Team, Pi of Sky, Chandra Team at McGill University, DFN, ATLAS Telescopes, High Time Resolution Universe Survey, RIMAS, RATIR, SKA South Africa/MeerKAT), Astrophys. J. Lett. 848, L12 (2017).

[26] F. Foucart, T. Hinderer, and S. Nissanke, Phys. Rev. D 98, 081501(R) (2018).

[27] K. Ackley, L. Amati, C. Barbieri, F. E. Bauer, S. Benetti, M. G. Bernardini, K. Bhirombhakdi, M. T. Botticella, M. Branchesi, E. Brocato et al., arXiv:2002.01950.

[28] C. J. Krüger and F. Foucart, Phys. Rev. D 101, 103002 (2020).

[29] L. Bildsten and C. Cutler, Astrophys. J. 400, 175 (1992).

[30] M. Vallisneri, Phys. Rev. Lett. 84, 3519 (2000).

[31] J. A. Faber, T. W. Baumgarte, S. L. Shapiro, K. Taniguchi, and F. A. Rasio, Phys. Rev. D 73, 024012 (2006).

[32] M. Shibata and K. Uryu, Class. Quantum Grav. 24, S125 (2007).

[33] Z. B. Etienne, Y. T. Liu, S. L. Shapiro, and T. W. Baumgarte, Phys. Rev. D 79, 044024 (2009).

[34] M. Shibata, K. Kyutoku, T. Yamamoto, and K. Taniguchi, Phys. Rev. D 79, 044030 (2009); 85, 127502(E) (2012).

[35] V. Ferrari, L. Gualtieri, and F. Pannarale, Phys. Rev. D 81, 064026 (2010).

[36] M. Shibata and K. Taniguchi, Living Rev. Relativ. 14, 6 (2011).

[37] F. Foucart, M. D. Duez, L. E. Kidder, and S. A. Teukolsky, Phys. Rev. D 83, 024005 (2011).

[38] K. Kyutoku, M. Shibata, and K. Taniguchi, Phys. Rev. D 82, 044049 (2010); 84, 049902(E) (2011).

[39] K. Kyutoku, H. Okawa, M. Shibata, and K. Taniguchi, Phys. Rev. D 84, 064018 (2011).

[40] F. Foucart, M. B. Deaton, M. D. Duez, L. E. Kidder, I. MacDonald, C. D. Ott, H. P. Pfeiffer, M. A. Scheel, B. Szilagyi, and S. A. Teukolsky, Phys. Rev. D 87, 084006 (2013).

[41] F. Foucart, M. D. Duez, L. E. Kidder, S. M. Nissanke, H. P. Pfeiffer, and M. A. Scheel, Phys. Rev. D 99, 103025 (2019). 
[42] T. A. Apostolatos, C. Cutler, G. J. Sussman, and K. S. Thorne, Phys. Rev. D 49, 6274 (1994).

[43] L. E. Kidder, Phys. Rev. D 52, 821 (1995).

[44] A. Vecchio, Phys. Rev. D 70, 042001 (2004).

[45] R. N. Lang and S. A. Hughes, Phys. Rev. D 74, 122001 (2006); 75, 089902(E) (2007); 77, 109901(E) (2008).

[46] A. Klein, P. Jetzer, and M. Sereno, Phys. Rev. D 80, 064027 (2009).

[47] K. Chatziioannou, N. Cornish, A. Klein, and N. Yunes, Astrophys. J. Lett. 798, L17 (2015).

[48] S. Vitale, R. Lynch, J. Veitch, V. Raymond, and R. Sturani, Phys. Rev. Lett. 112, 251101 (2014).

[49] R. O'Shaughnessy, B. Farr, E. Ochsner, H.-S. Cho, V. Raymond, C. Kim, and C.-H. Lee, Phys. Rev. D 89, 102005 (2014).

[50] V. Kalogera, Astrophys. J. 541, 319 (2000).

[51] I. Mandel and R. O'Shaughnessy, Class. Quantum Grav. 27, 114007 (2010).

[52] C. L. Rodriguez, M. Morscher, B. Pattabiraman, S. Chatterjee, C.-J. Haster, and F. A. Rasio, Phys. Rev. Lett. 115, 051101 (2015); 116, 029901(E) (2016).

[53] C. L. Rodriguez, M. Zevin, C. Pankow, V. Kalogera, and F. A. Rasio, Astrophys. J. Lett. 832, L2 (2016).

[54] W. M. Farr, S. Stevenson, M. Coleman Miller, I. Mandel, B. Farr, and A. Vecchio, Nature (London) 548, 426 (2017).

[55] B. Farr, D. E. Holz, and W. M. Farr, Astrophys. J. Lett. 854, L9 (2018).

[56] M. Fishbach, D. E. Holz, and B. Farr, Astrophys. J. Lett. 840, L24 (2017).

[57] K. Belczynski et al., Astron. Astrophys. 636, A104 (2020).

[58] D. Gerosa and E. Berti, Phys. Rev. D 95, 124046 (2017).

[59] C. Talbot and E. Thrane, Phys. Rev. D 96, 023012 (2017).

[60] X. Zhu, E. Thrane, S. Oslowski, Y. Levin, and P. D. Lasky, Phys. Rev. D 98, 043002 (2018).

[61] S. Stevenson, C. P. Berry, and I. Mandel, Mon. Not. R. Astron. Soc. 471, 2801 (2017).

[62] D. Wysocki, J. Lange, and R. O’Shaughnessy, Phys. Rev. D 100, 043012 (2019).

[63] B. Abbott et al. (LIGO Scientific Collaboration and Virgo Collaboration), Astrophys. J. Lett. 882, L24 (2019).

[64] C. Kimball, C. Talbot, C. P. Berry, M. Carney, M. Zevin, E. Thrane, and V. Kalogera, Astrophys. J. 900177 (2020).

[65] T. B. Littenberg, B. Farr, S. Coughlin, V. Kalogera, and D. E. Holz, Astrophys. J. Lett. 807, L24 (2015).

[66] C. Pankow, L. Sampson, L. Perri, E. Chase, S. Coughlin, M. Zevin, and V. Kalogera, Astrophys. J. 834, 154 (2017).

[67] H.-Y. Chen and K. Chatziioannou, Astrophys. J. Lett. 893, 2 (2019).

[68] M. Dominik, E. Berti, R. O’Shaughnessy, I. Mandel, K. Belczynski, C. Fryer, D. E. Holz, T. Bulik, and F. Pannarale, Astrophys. J. 806, 263 (2015).

[69] K. Belczynski, D. E. Holz, T. Bulik, and R. O'Shaughnessy, Nature (London) 534, 512 (2016).

[70] J. J. Eldridge and E. R. Stanway, Mon. Not. R. Astron. Soc. 462, 3302 (2016).

[71] S. Stevenson, A. Vigna-Gómez, I. Mandel, J. W. Barrett, C. J. Neijssel, D. Perkins, and S. E. de Mink, Nat. Commun. 8, 14906 (2017).

[72] S. F. Portegies Zwart and S. L. McMillan, Astrophys. J. 576, 899 (2002).
[73] K. Gultekin, M. Coleman Miller, and D. P. Hamilton, Astrophys. J. 640, 156 (2006).

[74] M. Mapelli, Mon. Not. R. Astron. Soc. 459, 3432 (2016).

[75] I. Bartos, B. Kocsis, Z. Haiman, and S. Márka, Astrophys. J. 835, 165 (2017).

[76] C. L. Rodriguez, C.-J. Haster, S. Chatterjee, V. Kalogera, and F. A. Rasio, Astrophys. J. Lett. 824, L8 (2016).

[77] S. Chatterjee, C. L. Rodriguez, V. Kalogera, and F. A. Rasio, Astrophys. J. Lett. 836, L26 (2017).

[78] M. Zevin, J. Samsing, C. Rodriguez, C.-J. Haster, and E. Ramirez-Ruiz, Astrophys. J. 871, 91 (2019).

[79] M. A. Sedda, Astrophys. J. 891, 47 (2020).

[80] B. McKernan, K. E. S. Ford, and R. O’Shaughnessy, Mon. Not. R. Astron. Soc. 498, 4088 (2020).

[81] S. Bird, I. Cholis, J. B. Muñoz, Y. Ali-Haïmoud, M. Kamionkowski, E. D. Kovetz, A. Raccanelli, and A. G. Riess, Phys. Rev. Lett. 116, 201301 (2016).

[82] S. Clesse and J. García-Bellido, Phys. Dark Univ. 15, 142 (2017).

[83] B. Abbott et al. (LIGO Scientific Collaboration and Virgo Collaboration), Astrophys. J. Lett. 818, L22 (2016).

[84] E. D. Kovetz, I. Cholis, P. C. Breysse, and M. Kamionkowski, Phys. Rev. D 95, 103010 (2017).

[85] M. Fishbach and D. E. Holz, Astrophys. J. Lett. 851, L25 (2017).

[86] C. Talbot and E. Thrane, Astrophys. J. 856, 173 (2018).

[87] B. Sathyaprakash, B. Schutz, and C. Van Den Broeck, Class. Quantum Grav. 27, 215006 (2010).

[88] B. Sathyaprakash et al., Class. Quantum Grav. 29, 124013 (2012); 30, 079501(E) (2013).

[89] S. R. Taylor and J. R. Gair, Phys. Rev. D 86, 023502 (2012).

[90] M. Dominik, K. Belczynski, C. Fryer, D. E. Holz, E. Berti, T. Bulik, I. Mandel, and R. O'Shaughnessy, Astrophys. J. 779, 72 (2013).

[91] C. L. Rodriguez and A. Loeb, Astrophys. J. Lett. 866, L5 (2018).

[92] M. Fishbach, D. E. Holz, and W. M. Farr, Astrophys. J. Lett. 863, L41 (2018).

[93] S. Vitale, W. M. Farr, K. Ng, and C. L. Rodriguez, Astrophys. J. Lett. 886, L1 (2019).

[94] M. Fishbach and D. E. Holz, Astrophys. J. Lett. 891, L27 (2020).

[95] U. N. Di Carlo, M. Mapelli, N. Giacobbo, M. Spera, Y. Bouffanais, S. Rastello, F. Santoliquido, M. Pasquato, A. Ballone, A. A. Trani et al., Mon. Not. R. Astron. Soc. 498, 495 (2020).

[96] A. S. Hamers and M. Safarzadeh, Astrophys. J. (unpublished).

[97] C. L. Rodriguez, M. Zevin, P. Amaro-Seoane, S. Chatterjee, K. Kremer, F. A. Rasio, and C. S. Ye, Phys. Rev. D 100, 043027 (2019).

[98] D. Gerosa, S. Vitale, and E. Berti, Phys. Rev. Lett. 125, 101103 (2020).

[99] M. Dominik, K. Belczynski, C. Fryer, D. Holz, E. Berti, T. Bulik, I. Mandel, and R. O'Shaughnessy, Astrophys. J. 759, 52 (2012)

[100] N. Giacobbo, M. Mapelli, and M. Spera, Mon. Not. R. Astron. Soc. 474, 2959 (2018).

[101] Y. Yang et al., Phys. Rev. Lett. 123, 181101 (2019).

[102] J. M. Lattimer, M. Prakash, D. Masak, and A. Yahil, Astrophys. J. 355, 241 (1990). 
[103] N. Chamel, P. Haensel, J. Zdunik, and A. Fantina, Int. J. Mod. Phys. E 22, 1330018 (2013).

[104] L. Rezzolla, E. R. Most, and L. R. Weih, Astrophys. J. Lett. 852, L25 (2018).

[105] B. Abbott et al. (LIGO Scientific Collaboration and Virgo Collaboration), Phys. Rev. Lett. 119, 161101 (2017).

[106] A. Gupta, D. Gerosa, K. G. Arun, E. Berti, W. M. Farr, and B. S. Sathyaprakash, Phys. Rev. D 101, 103036 (2020).

[107] T. A. Thompson et al., Science 366, 637 (2019).

[108] N. Giacobbo and M. Mapelli, Mon. Not. R. Astron. Soc. 480, 2011 (2018).

[109] D. Marković, Phys. Rev. D 48, 4738 (1993).

[110] C. Cutler and E. E. Flanagan, Phys. Rev. D 49, 2658 (1994).

[111] S. Nissanke, D. E. Holz, S. A. Hughes, N. Dalal, and J. L. Sievers, Astrophys. J. 725, 496 (2010).

[112] E. Baird, S. Fairhurst, M. Hannam, and P. Murphy, Phys. Rev. D 87, 024035 (2013).

[113] B. Barker and R. O’Connell, Phys. Rev. D 12, 329 (1975).

[114] K. S. Thorne and J. B. Hartle, Phys. Rev. D 31, 1815 (1985).

[115] É. Racine, Phys. Rev. D 78, 044021 (2008).

[116] P. Ajith et al., Phys. Rev. Lett. 106, 241101 (2011).

[117] L. Santamaria et al., Phys. Rev. D 82, 064016 (2010).

[118] P. Schmidt, F. Ohme, and M. Hannam, Phys. Rev. D 91, 024043 (2015).

[119] P. Schmidt, M. Hannam, and S. Husa, Phys. Rev. D 86, 104063 (2012).

[120] S. Fairhurst, R. Green, C. Hoy, M. Hannam, and A. Muir, Phys. Rev. D 102, 024055 (2020).

[121] S. Fairhurst, R. Green, M. Hannam, and C. Hoy, Phys. Rev. D 102, 041302 (2020).

[122] S. Ossokine, A. Buonanno, S. Marsat, R. Cotesta, S. Babak, T. Dietrich, R. Haas, I. Hinder, H. P. Pfeiffer, M. Pürrer et al., Phys. Rev. D 102, 044055 (2020).

[123] A. Buonanno and T. Damour, Phys. Rev. D 59, 084006 (1999).

[124] A. Buonanno and T. Damour, Phys. Rev. D 62, 064015 (2000).

[125] T. Damour, P. Jaranowski, and G. Schäfer, Phys. Rev. D 62, 084011 (2000).

[126] T. Damour, Phys. Rev. D 64, 124013 (2001).

[127] LIGO Scientific Collaboration and The Virgo Collaboration, GW190814 Data Release, https://doi.org/10.7935/zzw5-ak90 (2020).

[128] Y. Qin, T. Fragos, G. Meynet, J. Andrews, M. Sorensen, and H. Song, Astron. Astrophys. 616, A28 (2018).

[129] J. Fuller and L. Ma, Astrophys. J. Lett. 881, L1 (2019).

[130] S. Miller, T. A. Callister, and W. Farr, Astrophys. J. 895, 128 (2020).

[131] P. Schmidt, M. Hannam, S. Husa, and P. Ajith, Phys. Rev. D 84, 024046 (2011).

[132] B. Abbott et al. (KAGRA, LIGO Scientific, VIRGO), Living Rev. Relativ. 21, 3 (2018).

[133] R. T. Bayes, Philos. Trans. R. Soc. London 53, 370 (1764).

[134] J. Skilling, Bayesian Anal. 1, 833 (2006).

[135] J. Veitch and A. Vecchio, Phys. Rev. D 78, 022001 (2008).

[136] J. Veitch and A. Vecchio, Phys. Rev. D 81, 062003 (2010).

[137] J. Veitch et al., Phys. Rev. D 91, 042003 (2015).

[138] M. Tse et al., Phys. Rev. Lett. 123, 231107 (2019).
[139] F. Acernese et al. (Virgo Collaboration), Phys. Rev. Lett. 123, 231108 (2019).

[140] LIGO Scientific Collaboration and Virgo Collaboration, Noise curves used for simulations in the update of the observing scenarios paper, https://dcc.ligo.org/LIGO-T2000012/public (2019).

[141] S. Vitale, W. Del Pozzo, T. G. F. Li, C. Van Den Broeck, I. Mandel, B. Aylott, and J. Veitch, Phys. Rev. D 85, 064034 (2012).

[142] C. Cahillane et al. ( LIGO Scientific), Phys. Rev. D 96, 102001 (2017).

[143] L. Sun et al., Classical Quantum Gravity 51 (2020).

[144] S. Khan, S. Husa, M. Hannam, F. Ohme, M. Pürrer, X. Jiménez Forteza, and A. Bohé, Phys. Rev. D 93, 044007 (2016).

[145] S. Husa, S. Khan, M. Hannam, M. Pürrer, F. Ohme, X. J. Forteza, and A. Bohé, Phys. Rev. D 93, 044006 (2016).

[146] M. Hannam, P. Schmidt, A. Bohé, L. Haegel, S. Husa, F. Ohme, G. Pratten, and M. Pürrer, Phys. Rev. Lett. 113, 151101 (2014).

[147] J. Lange, R. O'Shaughnessy, and M. Rizzo, arXiv:1805.10457.

[148] S. Kullback and R. A. Leibler, Ann. Math. Stat. 22, 79 (1951).

[149] J. Lin, IEEE Trans. Inf. Theory 37, 145 (1991).

[150] R. E. Kass and A. E. Raftery, J. Am. Stat. Assoc. 90, 773 (1995).

[151] A. Gelman, J. B. Carlin, H. S. Stern, and D. B. Rubin, Bayesian Data Analysis, 2nd ed. (Chapman and Hall/CRC, Boca Raton, 2004).

[152] N. Cornish, L. Sampson, N. Yunes, and F. Pretorius, Phys. Rev. D 84, 062003 (2011).

[153] H.-S. Cho, E. Ochsner, R. O'Shaughnessy, C. Kim, and C.-H. Lee, Phys. Rev. D 87, 024004 (2013).

[154] E. Poisson and C. M. Will, Phys. Rev. D 52, 848 (1995).

[155] C. Hoy and V. Raymond, arXiv:2006.06639.

[156] B. Abbott et al. (LIGO Scientific Collaboration and Virgo Collaboration), Phys. Rev. X 9, 011001 (2019).

[157] D. Wysocki, R. O'Shaughnessy, L. Wade, and J. Lange, arXiv:2001.01747.

[158] F. Foucart, L. Buchman, M. D. Duez, M. Grudich, L. E. Kidder, I. MacDonald, A. Mroue, H. P. Pfeiffer, M. A. Scheel, and B. Szilagyi, Phys. Rev. D 88, 064017 (2013).

[159] B. D. Lackey, K. Kyutoku, M. Shibata, P. R. Brady, and J. L. Friedman, Phys. Rev. D 89, 043009 (2014).

[160] F. Pannarale, E. Berti, K. Kyutoku, B. D. Lackey, and M. Shibata, Phys. Rev. D 92, 084050 (2015).

[161] J. E. Thompson, E. Fauchon-Jones, S. Khan, E. Nitoglia, F. Pannarale, T. Dietrich, and M. Hannam, Phys. Rev. D 101, 124059 (2020).

[162] A. Matas et al., Phys. Rev. D 102, 043023 (2020).

[163] Y. Huang, C.-J. Haster, S. Vitale, V. Varma, F. Foucart, and S. Biscoveanu, arXiv:2005.11850.

[164] S. Vitale, R. Lynch, V. Raymond, R. Sturani, J. Veitch, and P. Graff, Phys. Rev. D 95, 064053 (2017).

[165] D. Trifirò, R. O’Shaughnessy, D. Gerosa, E. Berti, M. Kesden, T. Littenberg, and U. Sperhake, Phys. Rev. D 93, 044071 (2016).

[166] C. Afle et al., Phys. Rev. D 98, 083014 (2018).

[167] B. P. Abbott et al. (LIGO Scientific Collaboration and Virgo Collaboration), Class. Quantum Grav. 34, 104002 (2017). 
[168] R. Cotesta, A. Buonanno, A. Bohé, A. Taracchini, I. Hinder, and S. Ossokine, Phys. Rev. D 98, 084028 (2018).

[169] A. Nagar, G. Pratten, G. Riemenschneider, and R. Gamba, Phys. Rev. D 101, 024041 (2020).

[170] C. García-Quirós, M. Colleoni, S. Husa, H. Estellés, G. Pratten, A. Ramos-Buades, M. Mateu-Lucena, and R. Jaume, Phys. Rev. D 102, 064002 (2020).
[171] A. Nagar, G. Riemenschneider, G. Pratten, P. Rettegno, and F. Messina, Phys. Rev. D 102, 024077 (2020).

[172] G. Pratten, S. Husa, C. Garcia-Quiros, M. Colleoni, A. RamosBuades, H. Estelles, and R. Jaume, Phys. Rev. D 102, 064001 (2020).

[173] G. Pratten et al., arXiv:2004.06503. 